\title{
GLOBAL EXISTENCE, ASYMPTOTIC BEHAVIOR AND BLOW-UP OF SOLUTIONS FOR A SUSPENSION BRIDGE EQUATION WITH NONLINEAR DAMPING AND SOURCE TERMS
}

\author{
WENJUN LIU AND HEFENG ZHUANG
}

College of Mathematics and Statistics, Nanjing University of Information Science and Technol-ogy, NANJing 210044, China

E-mail address: wjliu@nuist.edu.cn (W. J. Liu); hfzhuang11@163.com (H. F. Zhuang)

\begin{abstract}
In this paper, we consider a fourth-order suspension bridge equation with nonlinear damping term $\left|u_{t}\right|^{m-2} u_{t}$ and source term $|u|^{p-2} u$. We give necessary and sufficient condition for global existence and energy decay results without considering the relation between $m$ and $p$. Moreover, when $p>m$, we give sufficient condition for finite time blow-up of solutions. The lower bound of the blow-up time $T_{\max }$ is also established. It worth to mention that our obtained results extend the recent results of Wang (J. Math. Anal. Appl., 2014) to the nonlinear damping case.
\end{abstract}

\section{INTRODUCTION}

Suspension bridge means a bridge takes the cable (or steel chain), which is hanged by tower and anchored on both sides (or both ends of the bridge), as an upper structure of the main load-bearing elements. Compared to other bridge structures, suspension bridge can use less material to span longer distance. Suspension bridge is suitable for the valley, rivers and other natural barrier regions. Due to these advantages, the construction method of suspension bridge is mostly used in modern bridges. At the same time, the security issues of suspension bridge have also attracted the attention of many scholars. Thus constructing a reasonable mathematical model and investigating the physical properties of suspension bridge are extremely necessary.

In this paper, we study the following fourth-order suspension bridge equation with nonlinear damping and source terms:

$$
\begin{cases}u_{t t}+\Delta^{2} u+a u+\left|u_{t}\right|^{m-2} u_{t}=|u|^{p-2} u, & (x, y, t) \in \Omega \times(0, T) \\ u(x, y, 0)=u_{0}(x, y), & (x, y) \in \bar{\Omega} \\ u_{t}(x, y, 0)=u_{1}(x, y), & (x, y) \in \bar{\Omega}\end{cases}
$$

with the boundary conditions

$$
\begin{cases}u(0, y, t)=u_{x x}(0, y, t)=u(\pi, y, t)=u_{x x}(\pi, y, t)=0, & (y, t) \in(-l, l) \times[0, T), \\ u_{y y}(x, \pm l, t)+\sigma u_{x x}(x, \pm l, t) & \\ =u_{y y y}(x, \pm l, t)+(2-\sigma) u_{x x y}(x, \pm l, t)=0, & (x, t) \in(0, \pi) \times[0, T),\end{cases}
$$

where $\Omega=(0, \pi) \times(-l, l) \subset \mathbb{R}^{2}, 2 \leq m<\infty, 2<p<\infty, 0<\sigma<\frac{1}{2}$ and $a=a(x, y)$ is a sign-changing and bounded measurable function. The initial data $u_{0}$ and $u_{1}$ belong to suitable spaces, which will be specified later.

There is a large number of publications concerning suspension bridge models. At the very beginning, in $[20,27,28]$, McKenna et al. suggested a one-dimensional simply supported beam suspended by hangers as a model for suspension bridges. It was assumed that when the hangers are stretched there is a restoring force, which is proportional to the amount of stretching; while when the beam moves in the opposite direction, the hangers slacken and there is no restoring force exerted on it. Let $u=u(x, t)$ be the vertical

2010 Mathematics Subject Classification. 35G31, 74K20, 35A01, 35B44.

Key words and phrases. Suspension bridges; fourth order wave equation; nonlinear damping; source term; existence; blow up 
displacement of the beam in the downward direction and $u^{+}=\max \{u, 0\}$, then the following fourth order nonlinear equation is derived:

$$
u_{t t}+u_{x x x x}+\gamma u^{+}=f(x, t), \quad x \in(0, L), t>0,
$$

where $\gamma u^{+}$represents the force due to the hangers, and $f$ is the forcing term acting on the bridge, including its own weight per unit length. For time periodic $f$, McKenna and Walter [27] proved the existence of multiple periodic solutions of problem (1.3).

However, when people study the collapse of Tacoma Narrows Bridge in 1940, they came to realize that it was not appropriate to simply consider the suspension bridge as a beam. The report [2] about the Tacoma Narrows Bridge collapse [36] concluded that "... the crucial event in the collapse to be the sudden change from a vertical to a torsional mode of oscillation". If one simply models a suspension bridge by a beam, there is no way to highlight the torsional oscillations. Therefore, a reliable model for suspension bridges should be nonlinear and it should have enough degrees of freedom to display torsional oscillations. By now, the nonlinear behavior of the suspension bridge is well established, see [1, 4, 11, 18, 35]. In [21], Lazer and McKenna suggested to study the following equation:

$$
\Delta^{2} u+c^{2} \Delta u+h(u)=0, \quad x \in \mathbb{R}^{n},
$$

where $h(u) \approx[u+1]^{+}-1$. After that, equations like (1.4) with the Navier boundary condition have been considered in many papers, see [22, 38, 41, 43]. Recently, Ferrero and Gazzola [8] suggested that one should consider the boundaries of plate $\Omega=(0, \pi) \times(-l, l)$ which represents the roadway of a suspension bridge as follows: due to the connection with the ground, the vertical edges of the plate is assumed to be hinged and thus

$$
u(0, y)=u_{x x}(0, y)=u(\pi, y)=u_{x x}(\pi, y)=0, \quad \forall y \in(-l, l) ;
$$

while the edges $y= \pm l$ are free and the boundary conditions at $y= \pm l$ become

$$
u_{y y}(x, \pm l)+\sigma u_{x x}(x, \pm l)=0, \quad u_{y y y}(x, \pm l)+(2-\sigma) u_{x x y}(x, \pm l)=0, \quad \forall x \in(0, \pi) .
$$

The free boundaries (1.6) yield small stretching energy for the plate, so Ferrero and Gazzola took $c=0$ in (1.4) and introduced the following model for the nonlinear dynamical suspension bridge:

$$
u_{t t}+\Delta^{2} u+\mu u_{t}+h(x, y, u)=f(x, y, t), \quad(x, y, t) \in \Omega \times(0, T),
$$

where $h(x, y, u)$ is restoring force due to the hangers of the suspension bridge, $f(x, y, t)$ is the external force including the gravity. More recently, given an open rectangular plate $\Omega=(0, \pi) \times(-l, l) \subset \mathbb{R}^{2}$, Wang [39] considered problem (1.1)-(1.2) but with a linear damping $(m=2)$. They proved the local existence, global existence and finite time blow-up of solutions as well as the estimation on the potential well depth. For more detail information about this suspension bridge model, we refer the readers to $[8,15]$ and references therein.

At the same time, the following wave equations with nonlinear damping and source terms have been extensively studied and many results concerning the existence and nonexistence were established:

$$
\begin{cases}u_{t t}-\Delta u+a\left|u_{t}\right|^{m-2} u_{t}=b|u|^{p-2} u, & (x, t) \in \Omega \times(0, T), \\ u(x, t)=0, & (x, t) \in \partial \Omega \times[0, T), \\ u(x, 0)=u_{0}(x), u_{t}(x, 0)=u_{1}(x), & x \in \Omega, t=0 .\end{cases}
$$

In the absence of damping term $(a=0)$, it is well known that the source term $b|u|^{p-2} u$ causes finite time blow-up of solutions with negative initial energy, see $[3,23,34]$. On the contrary, if there is no source term $(b=0)$, the damping term $a\left|u_{t}\right|^{m-2} u_{t}$ assures global existence for arbitrary initial data, see [13, 17]. After that, in $[23,24]$, Levine first considered the interaction between the damping and the source terms in the linear damping case $(m=2)$. He showed that solutions with negative initial energy blow up in finite time. Georgiev and Todorova [12] extended Levine's result to the nonlinear damping case $(m>2)$. In their work, the authors introduced a different method and determined suitable relations between $m$ and $p$ for which there is global existence or alternatively finite time blow-up. Specifically, they showed that solutions with any initial data continue to exist globally if $m \geq p$ and blow up in finite time if $p>m$ and the initial energy is sufficiently negative. Without imposing the condition that the initial energy is 
sufficiently negative, Messaoudi [29] extended the blow up result of [12] to solutions with negative initial energy only. It worth to mention that, in [14], without considering the relation between $m$ and $p$, the author established the global existence and finite time blow-up of solutions for problem (1.8) where $a>0$ and $b=1$ by using potential well theory. In [9], Guesmia studied the following equation:

$$
u_{t t}+\Delta^{2} u+q(x) u+g\left(u_{t}\right)=0
$$

where $q: \Omega \rightarrow R^{+}$is a bounded function. Under some assumptions, he showed the solution of (1.9) decays exponentially if $g$ behaves like a linear function, whereas the decay is polynomially otherwise. For more decay results, we refer the reader to $[5,6,7,10,16,19,25,26,37,40,44,45]$ and the references therein. In recent years, more authors pay attention to the lower and upper bounds for blow-up time. For example, Messaoudi [30] showed the upper bounds for blow-up time of a system of Petrovsky. Zhou [46] considered the lower bounds for blow-up time of the solutions to two nonlinear wave equations.

In the present paper, our purpose is to investigate the global existence, energy decay and finite time blow-up of solutions of initial-boundary value problem (1.1)-(1.2). We note here that au represents the restoring force because of the hangers of the suspension bridge, $\left|u_{t}\right|^{m-2} u_{t}$ describes the nonlinear internal friction and $|u|^{p-2} u$ represents the other external forces acting on the suspension bridge.

We pass to describe in more detail the remaining content of the paper.

In Section 2, we recall some materials given in [8] and [39], which are needed in our proofs.

In Section 3, for sake of convenience, we give two important lemmas as well as the detailed proofs.

In Section 4, we establish the existence and uniqueness of local solutions to (1.1)-(1.2) by using the contraction mapping principle.

In Section 5, without considering the relation between $m$ and $p$, we give necessary and sufficient condition for global existence and energy decay results. The proof of global existence result is based on the potential well theory and the continuous principle; while for energy decay result, the proof is based on the Nakao's inequality and some techniques given in [40].

In Section 6, under the condition that $p>m$, we give sufficient condition for blow-up result. We note that due to the presence of nonlinear damping term $\left|u_{t}\right|^{m-2} u_{t}$, it's difficult to apply the concavity method to our proof. So, our strategy is to construct an inequality for energy $E(t)$.

At last, in Section 7, by using the auxiliary functional introduced by Yang et al. [42], we establish the lower bound of the blow-up time $T_{\max }$.

It worth mentioning that our obtained results extend the recent results of Wang [39] to the nonlinear damping case. Compared to the results given in [39], the lower bound of the blow-up time $T_{\max }$ of the present paper is a new content.

\section{Preliminaries}

In this section, we recall some materials given in [8] and [39], which are needed in the proofs of our results.

Firstly, let $\Omega=(0, \pi) \times(-l, l) \subset \mathbb{R}^{2}$, we define the standard $L^{p}(\Omega)$ norm by $\|\cdot\|_{p}$ for $1 \leq p<\infty$ and the $H^{2}(\Omega)$ norm by $\|\cdot\|_{H^{2}}=\left(\|\cdot\|_{2}^{2}+\left\|D^{2} \cdot\right\|_{2}^{2}\right)^{\frac{1}{2}}$. In addition, we introduce the following functional space:

$$
H_{*}^{2}=H_{*}^{2}(\Omega):=\left\{u \in H^{2}(\Omega) \mid u=0 \text { on }\{0, \pi\} \times(-l, l)\right\} .
$$

The related dual space is defined by $\mathcal{H}(\Omega)$ and the corresponding duality between them is denoted by $\langle\cdot, \cdot\rangle$. It is easy to verify that $H_{*}^{2}$ satisfies $H_{0}^{2}(\Omega) \subset H_{*}^{2}(\Omega) \subset H^{2}(\Omega)$ and is a Hilbert space endowed with the inner product

$$
(u, v)_{H_{*}^{2}}=\int_{\Omega} \Delta u \Delta v d x d y+(1-\sigma) \int_{\Omega}\left(2 u_{x y} v_{x y}-u_{x x} v_{y y}-u_{y y} v_{x x}\right) d x d y, \quad \forall u, v \in H_{*}^{2} .
$$

This inner product induces a norm

$$
\|u\|_{H_{*}^{2}}=\left(\int_{\Omega}|\Delta u|^{2} d x d y+2(1-\sigma) \int_{\Omega}\left(u_{x y}^{2}-u_{x x} u_{y y}\right) d x d y\right)^{\frac{1}{2}}, \quad \forall u \in H_{*}^{2},
$$


which is equivalent to $\|\cdot\|_{H^{2}}$ for $0<\sigma<\frac{1}{2}$. For the proof we refer the reader to [8]. Moreover, we have the following Sobolev embedding inequality:

Lemma 2.1. [39] Assume that $1 \leq q<\infty$. Then for any $u \in H_{*}^{2}$, the inequality

$$
\|u\|_{q} \leq S_{q}\|u\|_{H_{*}^{2}}
$$

holds, where $S_{q}=\left(\frac{\pi}{2 l}+\frac{\sqrt{2}}{2}\right)(2 \pi l)^{\frac{q+2}{2 q}}\left(\frac{1}{1-\sigma}\right)^{\frac{1}{2}}$.

Remark 1. [39] Here $S_{q}$ is not less than the best Sobolev embedding constant. How to obtain the best constant is still open.

Besides, considering the following eigenvalue problem:

$$
\begin{cases}\Delta^{2} u=\Lambda u, & (x, y) \in \Omega, \\ u(0, y)=u_{x x}(0, y)=u(\pi, y)=u_{x x}(\pi, y)=0, & y \in(-l, l), \\ u_{y y}(x, \pm l)+\sigma u_{x x}(x, \pm l)=u_{y y y}(x, \pm l)+(2-\sigma) u_{x x y}(x, \pm l)=0, & x \in(0, \pi),\end{cases}
$$

we learn from [39] that the set of eigenvalues of (2.2) may be ordered in an increasing sequence $\left\{\Lambda_{i}\right\}_{i=1}^{\infty}$ of strictly positive numbers diverging to $+\infty$, and the least eigenvalue $\Lambda_{1}$ satisfies $0<\Lambda_{1}<1$. Also, we have the following basic results:

Lemma 2.2. [39] Assume that $-\Lambda_{1}<a_{1} \leq a \leq a_{2}$. Then for any $u \in H_{*}^{2}$, there holds

$$
A_{1}\|u\|_{H_{*}^{2}}^{2} \leq\|u\|_{H_{*}^{2}}^{2}+(a u, u)_{2} \leq A_{2}\|u\|_{H_{*}^{2}}^{2},
$$

where $(\cdot, \cdot)_{2}$ is the $L^{2}$ inner product and $A_{1}, A_{2}$ are given by

$$
A_{1}= \begin{cases}1+\frac{a_{1}}{\Lambda_{1}}, & a_{1}<0 \\ 1, & a_{1} \geq 0\end{cases}
$$

and

$$
A_{2}= \begin{cases}1, & a_{2}<0 \\ 1+\frac{a_{2}}{\Lambda_{1}}, & a_{2} \geq 0\end{cases}
$$

Now, we define the Nehari functional $I$ and the energy functional $J$ :

$$
\begin{aligned}
& I(u)=\|u\|_{H_{*}^{2}}^{2}+(a u, u)_{2}-\|u\|_{p}^{p}, \quad \text { for every } u \in H_{*}^{2}, \\
& J(u)=\frac{1}{2}\|u\|_{H_{*}^{2}}^{2}+\frac{1}{2}(a u, u)_{2}-\frac{1}{p}\|u\|_{p}^{p}, \quad \text { for every } u \in H_{*}^{2},
\end{aligned}
$$

which play critical roles in dealing with our problem. Let $u$ be an arbitrary nonzero element in $H_{*}^{2}$ and consider a real value function defined by

$$
j(\lambda)=J(\lambda u), \quad \lambda \geq 0,
$$

then

$$
\begin{gathered}
j^{\prime}(\lambda)=\lambda\|u\|_{H_{*}^{2}}^{2}+\lambda(a u, u)_{2}-\lambda^{p-1}\|u\|_{p}^{p}, \\
j^{\prime \prime}(\lambda)=\|u\|_{H_{*}^{2}}^{2}+(a u, u)_{2}-(p-1) \lambda^{p-2}\|u\|_{p}^{p} .
\end{gathered}
$$

Clearly, $j(0)=j^{\prime}(0)=0$ and $j^{\prime \prime}(0)=\|u\|_{H_{*}^{2}}^{2}+(a u, u)_{2}>0$ for $a>-\Lambda_{1}$. Thus for any $0 \neq u \in H_{*}^{2}, j(\lambda)$ is a convex function for small $\lambda>0$ and has the following behaviors:

Lemma 2.3. [39] Assume that $-\Lambda_{1}<a_{1} \leq a \leq a_{2}$. Then for any nontrivial $u \in H_{*}^{2}$,

(i) $\lim _{\lambda \rightarrow \infty} j(\lambda)=-\infty$;

(ii) there exists a unique $\bar{\lambda}=\bar{\lambda}(u)>0$ such that $j^{\prime}(\bar{\lambda})=0$;

(iii) $j^{\prime \prime}(\bar{\lambda})<0$. 
Then, we could define the potential well depth of the functional $J$ (also known as mountain pass level) by

$$
d=\inf _{u \in H_{*}^{2} \backslash\{0\}} \max _{\lambda>0} J(\lambda u) .
$$

Denote the set of all nontrivial stationary solutions to the problem (1.1)-(1.2) by

$$
N=\left\{u \in H_{*}^{2} \backslash\{0\}: I(u)=0\right\},
$$

which is the so-called Nehari manifold. By considering a map $s \mapsto I(s u)$ for all $u$ such that $\|u\|_{H_{*}^{2}}^{2}=1$ and Lemma 2.3, it is easy to check that each half line starting from the origin of $H_{*}^{2}$ intersects only once the manifold $N$ and $N$ separates the following two sets:

$$
N_{+}=\left\{u \in H_{*}^{2}: I(u)>0\right\} \cup\{0\} \quad \text { and } \quad N_{-}=\left\{u \in H_{*}^{2}: I(u)<0\right\} .
$$

Then the stable set $W$ and unstable set $U$ may be defined by

$$
W=\left\{u \in N_{+}: J(u)<d\right\}, \quad U=\left\{u \in N_{-}: J(u)<d\right\}
$$

Lemma 2.4. [39] The following properties of $W$ and $U$ hold:

(i) $W$ is a neighborhood of $0 \in H_{*}^{2}$;

(ii) $0 \notin \bar{U}$ (closure in $H_{*}^{2}$ )

As Payne and Sattinger did in [34], the potential well depth $d$ defined in (2.6) can be also characterized as

$$
d=\inf _{u \in N} J(u) .
$$

Finally, we consider an energy functional $\mathcal{E}: H_{*}^{2}(\Omega) \times L^{2}(\Omega) \rightarrow \mathbb{R}$ defined by

$$
\mathcal{E}(v, w)=J(v)+\frac{1}{2}\|w\|_{2}^{2}, \quad \text { for every pair }(v, w) \in H_{*}^{2}(\Omega) \times L^{2}(\Omega),
$$

and the Lyapunov function $E(t)=\mathcal{E}\left(u(t), u_{t}(t)\right)$, defined for any solution $u(t)$ of problem (1.1)-(1.2). That is

$$
E(t)=\frac{1}{2}\|u(t)\|_{H_{*}^{2}}^{2}+\frac{1}{2}\left\|u_{t}(t)\right\|_{2}^{2}+\frac{1}{2}(a u(t), u(t))_{2}-\frac{1}{p}\|u(t)\|_{p}^{p},
$$

which satisfies that

$$
E(t)+\int_{s}^{t}\left\|u_{\tau}(\tau)\right\|_{m}^{m} d \tau=E(s), \quad \text { for every } 0 \leq s \leq t<T_{\max } .
$$

\section{Some calculations}

In this section, we introduce two lemmas, which will be used in the following paper.

Lemma 3.1. [31] For $u, v \in H^{4}(\Omega) \cap H_{*}^{2}(\Omega)$ satisfying (1.2), we have

$$
\left(\Delta^{2} u, v\right)_{L^{2}(\Omega)}=(u, v)_{H_{*}^{2}(\Omega)} .
$$

Remark 2. We note that this lemma is given in many literatures, but it is rare to see its proof. Here we give a detailed proof of it.

Proof. Note first that, for all $u \in H^{4}(\Omega) \cap H_{*}^{2}(\Omega)$ satisfying (1.2), we have

$$
u(0, y)=u(\pi, y)=u_{y}(0, y)=u_{y}(\pi, y)=u_{y y}(0, y)=u_{y y}(\pi, y)=0
$$


for any $y \in(-l, l)$. Then, by adapting the Gauss-Green formula and the definition of $(u, v)_{H_{*}^{2}}$, we have

$$
\begin{aligned}
& \int_{\Omega} \Delta^{2} u v d x d y-(u, v)_{H_{*}^{2}} \\
= & \int_{\Omega} \Delta u \Delta v d x d y-\int_{\partial \Omega}\left[\Delta u v_{\nu}-v(\Delta u)_{\nu}\right] d s-\int_{\Omega} \Delta u \Delta v d x d y \\
& -(1-\sigma) \int_{\Omega}\left(2 u_{x y} v_{x y}-u_{x x} v_{y y}-u_{y y} v_{x x}\right) d x d y \\
= & -\left\{\int_{\partial \Omega}\left[\Delta u v_{\nu}-v(\Delta u)_{\nu}\right] d s+(1-\sigma) \int_{\Omega}\left(2 u_{x y} v_{x y}-u_{x x} v_{y y}-u_{y y} v_{x x}\right) d x d y\right\}
\end{aligned}
$$

Next, we prove that

$$
\begin{aligned}
& \int_{\partial \Omega}\left[\Delta u v_{\nu}-v(\Delta u)_{\nu}\right] d s+(1-\sigma) \int_{\Omega}\left(2 u_{x y} v_{x y}-u_{x x} v_{y y}-u_{y y} v_{x x}\right) d x d y=0 . \\
& \int_{\partial \Omega}\left[\Delta u v_{\nu}-v(\Delta u)_{\nu}\right] d s+(1-\sigma) \int_{\Omega}\left(2 u_{x y} v_{x y}-u_{x x} v_{y y}-u_{y y} v_{x x}\right) d x d y \\
& =\int_{-l}^{l}\left[u_{x x}(\pi, y) v_{x}(\pi, y)-u_{x x}(0, y) v_{x}(0, y)\right] d y \\
& +\int_{0}^{\pi}\left\{\left[u_{y y y}(x,-l)+(2-\sigma) u_{x x y}(x,-l)\right] v(x,-l)-\left[u_{y y}(x,-l)+\sigma u_{x x}(x,-l)\right] v_{y}(x,-l)\right\} d x \\
& +\int_{0}^{\pi}\left\{\left[u_{y y}(x, l)+\sigma u_{x x}(x, l)\right] v_{y}(x, l)-\left[u_{y y y}(x, l)+(2-\sigma) u_{x x y}(x, l)\right] v(x, l)\right\} d x \\
& +(1-\sigma) \int_{\Omega}\left(2 u_{x y} v_{x y}-u_{x x} v_{y y}-u_{y y} v_{x x}\right) d x d y \\
& -\left\{\int_{0}^{\pi}\left[(1-\sigma) u_{x x y}(x,-l) v(x,-l)-(\sigma-1) u_{x x}(x,-l) v_{y}(x,-l)\right] d x\right. \\
& \left.+\int_{0}^{\pi}\left[(\sigma-1) u_{x x}(x, l) v_{y}(x, l)-(1-\sigma) u_{x x y}(x, l) v(x, l)\right] d x\right\} .
\end{aligned}
$$

We note that

$$
\begin{aligned}
& (1-\sigma) \int_{\Omega}\left(2 u_{x y} v_{x y}-u_{x x} v_{y y}-u_{y y} v_{x x}\right) d x d y \\
& -\left\{\int_{0}^{\pi}\left[(1-\sigma) u_{x x y}(x,-l) v(x,-l)-(\sigma-1) u_{x x}(x,-l) v_{y}(x,-l)\right] d x\right. \\
& \left.+\int_{0}^{\pi}\left[(\sigma-1) u_{x x}(x, l) v_{y}(x, l)-(1-\sigma) u_{x x y}(x, l) v(x, l)\right] d x\right\} \\
= & (1-\sigma) \int_{\Omega}\left(2 u_{x y} v_{x y}-u_{x x} v_{y y}-u_{y y} v_{x x}\right) d x d y+(1-\sigma) \int_{0}^{\pi} \int_{-l}^{l} \frac{\partial\left(u_{x x y} v+u_{x x} v_{y}\right)}{\partial y} d y d x \\
= & (1-\sigma)\left\{\int_{\Omega}\left(2 u_{x y} v_{x y}-u_{x x} v_{y y}-u_{y y} v_{x x}+u_{x x y y} v+u_{x x y} v_{y}+u_{x x y} v_{y}+u_{x x} v_{y y}\right) d x d y\right\} \\
= & (1-\sigma)\left\{2 \int_{-l}^{l} \int_{0}^{\pi}\left(u_{x y} v_{x y}+u_{x x y} v_{y}\right) d x d y+\int_{-l}^{l} \int_{0}^{\pi}\left(u_{x x y y} v-u_{y y} v_{x x}\right) d x d y\right\} .
\end{aligned}
$$

We find that

$$
2 \int_{-l}^{l} \int_{0}^{\pi}\left(u_{x y} v_{x y}+u_{x x y} v_{y}\right) d x d y=\left.2 \int_{-l}^{l} u_{x y} v_{y}\right|_{0} ^{\pi} d y=0
$$


and

$$
\begin{aligned}
& \int_{-l}^{l} \int_{0}^{\pi} u_{y y} v_{x x} d x d y=\int_{-l}^{l}\left\{\left.u_{y y} v_{x}\right|_{0} ^{\pi}-\int_{0}^{\pi} v_{x} u_{x y y} d x\right\} d y \\
= & -\int_{-l}^{l} \int_{0}^{\pi} v_{x} u_{x y y} d x d y=-\int_{-l}^{l}\left\{\left.v u_{x y y}\right|_{0} ^{\pi}-\int_{0}^{\pi} u_{x x y y} v d x\right\} d y=\int_{-l}^{l} \int_{0}^{\pi} u_{x x y y} v d x d y .
\end{aligned}
$$

Thus

$$
\begin{aligned}
& (1-\sigma) \int_{\Omega}\left(2 u_{x y} v_{x y}-u_{x x} v_{y y}-u_{y y} v_{x x}\right) d x d y \\
& -\left\{\int_{0}^{\pi}\left[(1-\sigma) u_{x x y}(x,-l) v(x,-l)-(\sigma-1) u_{x x}(x,-l) v_{y}(x,-l)\right] d x\right. \\
& \left.+\int_{0}^{\pi}\left[(\sigma-1) u_{x x}(x, l) v_{y}(x, l)-(1-\sigma) u_{x x y}(x, l) v(x, l)\right] d x\right\} \\
& =0
\end{aligned}
$$

which combining with the boundary conditions (1.2) leads to

$$
\int_{\partial \Omega}\left[\Delta u v_{\nu}-v(\Delta u)_{\nu}\right] d s+(1-\sigma) \int_{\Omega}\left(2 u_{x y} v_{x y}-u_{x x} v_{y y}-u_{y y} v_{x x}\right) d x d y=0
$$

That is

$$
\left(\Delta^{2} u, v\right)_{L^{2}(\Omega)}=\int_{\Omega} \Delta^{2} u v d x d y=(u, v)_{H_{*}^{2}(\Omega)}
$$

The proof is completed.

Lemma 3.2. For $u \in H_{*}^{2}(\Omega)$ satisfying (1.2), we have

$$
\int_{t_{1}}^{t_{2}}\left(u, u_{\tau}\right)_{H_{*}^{2}} d \tau=\frac{1}{2}\left\|u\left(t_{2}\right)\right\|_{H_{*}^{2}}^{2}-\frac{1}{2}\left\|u\left(t_{1}\right)\right\|_{H_{*}^{2}}^{2}
$$

Proof. Using the definition of $\left(u, u_{t}\right)_{H_{*}^{2}}$ directly, we can get

$$
\begin{aligned}
& \int_{t_{1}}^{t_{2}}\left(u, u_{\tau}\right)_{H_{*}^{2}} d \tau \\
= & \int_{t_{1}}^{t_{2}} \int_{\Omega}\left[\Delta u \Delta u_{\tau}+(1-\sigma)\left(2 u_{x y} u_{x y \tau}-u_{x x} u_{y y \tau}-u_{y y} u_{x x \tau}\right)\right] d x d y d \tau \\
= & \int_{\Omega}\left\{\int_{t_{1}}^{t_{2}} \Delta u d(\Delta u(\cdot, \tau))+(1-\sigma)\left(2 \int_{t_{1}}^{t_{2}} u_{x y} d\left(u_{x y}(\cdot, \tau)\right)-\int_{t_{1}}^{t_{2}} d\left(u_{x x}(\cdot, \tau) u_{y y}(\cdot, \tau)\right)\right)\right\} d x d y \\
= & \int_{\Omega}\left\{\left.\frac{\Delta u \Delta u}{2}\right|_{t_{1}} ^{t_{2}}+(1-\sigma)\left(2 \times\left.\frac{u_{x y} u_{x y}}{2}\right|_{t_{1}} ^{t_{2}}-\left.\frac{2 u_{x x} u_{y y}}{2}\right|_{t_{1}} ^{t_{2}}\right)\right\} d x d y \\
= & \frac{1}{2}\left\|u\left(t_{2}\right)\right\|_{H_{*}^{2}}^{2}-\frac{1}{2}\left\|u\left(t_{1}\right)\right\|_{H_{*}^{2}}^{2} .
\end{aligned}
$$

The proof is completed.

\section{Local existence}

In this section, we are concerned with the local existence of solutions to problem (1.1)-(1.2). To begin with, we explain what does a weak solution mean to our problem.

Definition 1. A function $u \in C\left([0, T], H_{*}^{2}(\Omega)\right) \cap C^{1}\left([0, T], L^{2}(\Omega)\right) \cap C^{2}([0, T], \mathcal{H}(\Omega))$ with $u_{t} \in L^{m}([0, T]$, $\left.L^{m}(\Omega)\right)$ is called a weak solution to (1.1)-(1.2), if the following conditions hold

$$
u(0)=u_{0}, \quad u_{t}(0)=u_{1}
$$


and

$$
\left\langle u_{t t}, \eta\right\rangle+(u, \eta)_{H_{*}^{2}}+\int_{\Omega} a u \eta d x d y+\int_{\Omega}\left|u_{t}\right|^{m-2} u_{t} \eta d x d y=\int_{\Omega}|u|^{p-2} u \eta d x d y
$$

for all $\eta \in H_{*}^{2}(\Omega)$ and a.e. $t \in[0, T]$.

Then, we have the following local existence theorem.

Theorem 4.1. Suppose that $m \geq 2, p>2$ and $-\Lambda_{1}<a_{1} \leq a \leq a_{2}$ hold. The initial data $\left(u_{0}, u_{1}\right) \in$ $H_{*}^{2}(\Omega) \times L^{2}(\Omega)$ are given. Then problem (1.1)-(1.2) admits a unique local weak solution for some $T>0$ such that

$$
\begin{aligned}
& u \in C\left([0, T], H_{*}^{2}(\Omega)\right) \cap C^{1}\left([0, T], L^{2}(\Omega)\right) \cap C^{2}([0, T], \mathcal{H}(\Omega)), \\
& u_{t} \in C\left([0, T], L^{2}(\Omega)\right) \cap L^{m}([0, T] \times \Omega) .
\end{aligned}
$$

Moreover, if

$$
T_{\text {max }}=\sup \{T>0: u=u(t) \text { exists on }[0, T]\}<\infty,
$$

then

$$
\lim _{t \rightarrow T_{\max }}\|u(t)\|_{q}=\infty, \text { for } q \geq 1 \text { such that } q>\frac{p-2}{2} .
$$

Proof. We start with some definitions. For every $T>0$, we set the space

$$
H=C\left([0, T], H_{*}^{2}(\Omega)\right) \cap C^{1}\left([0, T], L^{2}(\Omega)\right)
$$

with the norm

$$
\|u(t)\|_{H}=\left(\max _{t \in[0, T]}\left(A_{1}\|u(t)\|_{H_{*}^{2}(\Omega)}^{2}+\left\|u_{t}(t)\right\|_{2}^{2}\right)\right)^{\frac{1}{2}},
$$

where $A_{1}$ is given in Lemma 2.2. For $u_{0} \in H_{*}^{2}(\Omega), u_{1} \in L^{2}(\Omega)$, we denote

$$
M_{T}=\left\{u \in H: u(0)=u_{0}, u_{t}(0)=u_{1} \text { and }\|u(t)\|_{H}^{2} \leq R^{2}\right\},
$$

where $R^{2} \geq 2\left(A_{2}\left\|u_{0}\right\|_{H_{*}^{2}}^{2}+\left\|u_{1}\right\|_{2}^{2}\right)$ and $A_{2}$ is given in Lemma 2.2 .

Then we consider the initial problem

$$
\begin{cases}v_{t t}+\Delta^{2} v+a v+\left|v_{t}\right|^{m-2} v_{t}=|u|^{p-2} u, & (x, y, t) \in \Omega \times(0, T) \\ v(x, y, 0)=u_{0}(x, y), & (x, y) \in \bar{\Omega} \\ v_{t}(x, y, 0)=u_{1}(x, y), & (x, y) \in \bar{\Omega}\end{cases}
$$

with the boundary conditions

$$
\begin{cases}v(0, y, t)=v_{x x}(0, y, t)=v(\pi, y, t)=v_{x x}(\pi, y, t)=0, & (y, t) \in(-l, l) \times[0, T), \\ v_{y y}(x, \pm l, t)+\sigma v_{x x}(x, \pm l, t) & \\ =v_{y y y}(x, \pm l, t)+(2-\sigma) v_{x x y}(x, \pm l, t)=0, & (x, t) \in(0, \pi) \times[0, T),\end{cases}
$$

for every $t \in[0, T]$.

Lemma 4.1. Assume that $u_{0} \in H_{*}^{2}(\Omega), u_{1} \in L^{2}(\Omega)$ and $-\Lambda_{1}<a_{1} \leq a \leq a_{2}$. Then for every $u \in H$, there exists a unique solution $v \in H \cap C^{2}([0, T], \mathcal{H}(\Omega))$ with $v_{t} \in L^{m}([0, T] \times \Omega)$ to the problem (4.1)-(4.2).

Proof. According to [8, Theorem 3.6] and [39, Lemma 4.1], we can easily prove that there exists a $v \in H \cap C^{2}([0, T], \mathcal{H}(\Omega))$ satisfies problem (4.1)-(4.2). To complete the proof, here we need to prove that $v_{t} \in L^{m}([0, T] \times \Omega)$.

Taking $v_{t}$ as a test function and integrate over $\Omega \times[0, t] \subset \Omega \times[0, T]$, we have

$$
\begin{aligned}
& \int_{0}^{t}\left\langle v_{\tau \tau}(\tau), v_{\tau}(\tau)\right\rangle d \tau+\int_{0}^{t}\left(v(\tau), v_{\tau}(\tau)\right)_{H_{*}^{2}} d \tau+\int_{0}^{t}\left\|v_{\tau}(\tau)\right\|_{m}^{m} d \tau+\int_{0}^{t}\left(a v(\tau), v_{\tau}(\tau)\right)_{2} d \tau \\
= & \int_{0}^{t} \int_{\Omega}|u(\tau)|^{p-2} u(\tau) v_{\tau}(\tau) d x d y d \tau .
\end{aligned}
$$


Recalling that $a_{1} \leq a \leq a_{2}$ and by Lemma 2.2 and 3.2 , we have

$$
\begin{aligned}
& \left\|v_{t}(t)\right\|_{2}^{2}+A_{1}\|v(t)\|_{H_{*}^{2}}^{2}+2 \int_{0}^{t}\left\|v_{\tau}(\tau)\right\|_{m}^{m} d \tau \\
\leq & \left\|u_{1}\right\|_{2}^{2}+A_{2}\left\|u_{0}\right\|_{H_{*}^{2}}^{2}+2 \int_{0}^{t} \int_{\Omega}|u(\tau)|^{p-2} u(\tau) v_{\tau}(\tau) d x d y d \tau .
\end{aligned}
$$

Notice that

$$
\begin{aligned}
2 \int_{0}^{t} \int_{\Omega}|u(\tau)|^{p-2} u(\tau) v_{\tau}(\tau) d x d y d \tau & =2 \int_{0}^{t} \int_{\Omega}|u(\tau)|^{p-1} v_{\tau}(\tau) d x d y d \tau \\
& \leq 2 \int_{0}^{t}\|u(\tau)\|_{\frac{(p-1) m}{m-1}}^{p-1}\left\|v_{\tau}(\tau)\right\|_{m} d \tau \\
& \leq 2 C(\varepsilon) \int_{0}^{t}\|u(\tau)\|_{\frac{(p-1) m}{m-1}}^{\frac{(p-1)}{m-1}} d \tau+2 \varepsilon \int_{0}^{t}\left\|v_{\tau}(\tau)\right\|_{m}^{m} d \tau \\
& \leq 2 C(\varepsilon) \int_{0}^{t}\|u(\tau)\|_{H_{*}^{2}}^{\frac{(p-1) m}{m-1}} d \tau+2 \varepsilon \int_{0}^{t}\left\|v_{\tau}(\tau)\right\|_{m}^{m} d \tau \\
& \leq C T+2 \varepsilon \int_{0}^{t}\left\|v_{\tau}(\tau)\right\|_{m}^{m} d \tau
\end{aligned}
$$

Taking $\varepsilon$ small enough and from (4.3), we have

$$
\int_{0}^{t}\left\|v_{\tau}(\tau)\right\|_{m}^{m} d \tau \leq C T
$$

which deduces that $v_{t} \in L^{m}([0, T] \times \Omega)$ and completes the proof of Lemma 4.1.

Now, we come to prove Theorem 4.1. For any $u \in M_{T}$, we defined $v=\Phi(u)$ such that $v$ is the solution to problem (4.1)-(4.2). Then Lemma 4.1 shows that $\Phi$ is well defined and it maps $H$ into $H$. We prove that, for some small $T>0$,

(i) $\Phi$ maps a ball $B_{R}$ of radius $R$ in $M_{T}$ into itself;

(ii) $\Phi$ is a contraction in $B_{R}$.

In fact, assume that $u \in M_{T}$, then the corresponding solution $v=\Phi(u)$ satisfies (4.3) for all $t \in[0, T]$. Thus, as we did in the proof of Lemma 4.1, there holds

$$
\begin{aligned}
\left\|v_{t}(t)\right\|_{2}^{2}+A_{1}\|v(t)\|_{H_{*}^{2}}^{2} & \leq\left\|u_{1}\right\|_{2}^{2}+A_{2}\left\|u_{0}\right\|_{H_{*}^{2}}^{2}+C R^{\frac{(p-1) m}{m-1}} T \\
& \leq \frac{R^{2}}{2}+C R^{\frac{(p-1) m}{m-1}} T .
\end{aligned}
$$

If $T$ is small enough, then $\|v\|_{H} \leq R$, which implies that $\Phi\left(M_{T}\right) \subseteq M_{T}$.

To check (ii), we set $v_{1}=\Phi\left(w_{1}\right), v_{2}=\Phi\left(w_{2}\right)$ with $w_{1}, w_{2} \in M_{T}$. Putting $v_{1}, v_{2}$ in Eq. (4.1) and then subtracting the two equations, we obtain by setting $v=v_{1}-v_{2}$

$$
\begin{aligned}
& \left\langle v_{t t}, \eta\right\rangle+(v, \eta)_{H_{*}^{2}}+(a v, \eta)_{2}+\int_{\Omega}\left(\left|v_{1 t}\right|^{m-2} v_{1 t}-\left|v_{2 t}\right|^{m-2} v_{2 t}\right) \eta d x d y \\
= & \int_{\Omega}\left(\left|w_{1}\right|^{p-2} w_{1}-\left|w_{2}\right|^{p-2} w_{2}\right) \eta d x d y \\
= & \int_{\Omega} \gamma(t)\left(w_{1}-w_{2}\right) \eta d x d y
\end{aligned}
$$

for all $\eta \in H_{*}^{2}$ and a.e. $t \in[0, T]$, where $\gamma(t)$ is estimated by $\gamma(t) \leq(p-1)\left(\left|w_{1}\right|+\left|w_{2}\right|\right)^{p-2}$.

Taking $\eta=v_{t}=v_{1 t}-v_{2 t}$, arguing similarly as above and noting that

$$
\int_{\Omega}\left(\left|v_{1 t}\right|^{m-2} v_{1 t}-\left|v_{2 t}\right|^{m-2} v_{2 t}\right)\left(v_{1 t}-v_{2 t}\right) d x d y \geq 0,
$$


we have

$$
\left\|\Phi\left(w_{1}\right)-\Phi\left(w_{2}\right)\right\|_{H}^{2}=\|v\|_{H}^{2} \leq C R^{2 p-4} T\left\|w_{1}-w_{2}\right\|_{H}^{2} .
$$

Likely, if $T$ is so small that $C R^{2 p-4} T<1$, then there exists a constant $0<\delta<1$ such that

$$
\left\|\Phi\left(w_{1}\right)-\Phi\left(w_{2}\right)\right\|_{H}^{2} \leq \delta\left\|w_{1}-w_{2}\right\|_{H}^{2} .
$$

Therefore, $\Phi$ is a contract map and (ii) is proved.

According to the contraction mapping principle, there exists $u \in M_{T}$ such that $u=\Phi(u)$, which is a solution to problem (4.1)-(4.2). Using (4.4) again, we can easily prove the uniqueness of the solution.

Finally, by the continue principle, we know that if $\|u(t)\|_{H}<\infty$, the solution $u(t)$ should be continued, see also [33, p. 158] for a similar argument. Hence, if $T_{\max }<\infty$, it follows

$$
\lim _{t \rightarrow T_{\max }}\left(A_{1}\|u(t)\|_{H_{*}^{2}}^{2}+\left\|u_{t}(t)\right\|_{2}^{2}\right)=\lim _{t \rightarrow T_{\max }}\|u(t)\|_{H}^{2}=\infty .
$$

From (2.8) and (2.9), we know that $E(t)$ is nonincreasing and

$$
\frac{1}{2}\|u(t)\|_{H_{*}^{2}}^{2}+\frac{1}{2}\left\|u_{t}(t)\right\|_{2}^{2}+\frac{1}{2}(a u(t), u(t))_{2} \leq \frac{1}{p}\|u(t)\|_{p}^{p}+E(0), \quad \text { for all } t \in\left[0, T_{\max }\right) .
$$

By Lemma 2.2, there holds

$$
\frac{A_{1}}{2}\|u(t)\|_{H_{*}^{2}}^{2}+\frac{1}{2}\left\|u_{t}(t)\right\|_{2}^{2} \leq \frac{1}{p}\|u(t)\|_{p}^{p}+E(0), \quad \text { for all } t \in\left[0, T_{\max }\right) .
$$

According to (4.5), it implies that

By Lemma 2.1, we have

$$
\lim _{t \rightarrow T_{\max }}\|u(t)\|_{p}=\infty
$$

$$
\lim _{t \rightarrow T_{\max }}\|u(t)\|_{H_{*}^{2}}=\infty
$$

Moreover, by (4.6)

$$
\frac{A_{1}}{2}\|u(t)\|_{H_{*}^{2}}^{2} \leq \frac{1}{p}\|u(t)\|_{p}^{p}+E(0),
$$

which combined with Gagliardo-Nirenberg inequality yields that

$$
C\|u(t)\|_{H_{*}^{2}}^{2}-C \leq\|u(t)\|_{p}^{p} \leq C\|u(t)\|_{q}^{p(1-\alpha)}\|u(t)\|_{H_{*}^{2}}^{p \alpha}, \quad \text { for } \alpha=\frac{2(p-q)}{p(q+2)} .
$$

If $\alpha \in(0,1)$ such that $p \alpha<2$, that is $\frac{p-2}{2}<q<p$, then the above inequality and (4.7) immediately yield

$$
\lim _{t \rightarrow T_{\max }}\|u(t)\|_{q}=\infty, \quad \text { for } q \geq 1 \text { such that } q>\frac{p-2}{2} .
$$

The proof is completed.

\section{Global existence And energy decay}

In this section, we state and prove the necessary and sufficient conditions for global existence and energy decay of solutions to problem (1.1)-(1.2). We need to estimate the potential well depth first.

Lemma 5.1. [39] Assume that $-\Lambda_{1}<a_{1} \leq a \leq a_{2}, 2<p<\infty$. Then the potential well depth $d$ can be estimated by

$$
0<D=\frac{p-2}{2 p} A_{1}^{\frac{p}{p-2}} S_{p}^{-\frac{2 p}{p-2}} \leq d \leq \frac{p-2}{2 p} C_{p}
$$

where $S_{p}$ is the Sobolev embedding constant, $A_{1}$ is defined in Lemma 2.2 and $C_{p}$ is given by

$$
C_{p}=\left(\frac{\pi\left(1+a_{2}\right)}{2\|\sin x\|_{p}^{2}}\right)^{\frac{p}{p-2}} .
$$

In addition, we need to introduce the following lemma which will be used in our proof. 
Lemma 5.2. [32] Let $\phi(t)$ be a nonincreasing and nonnegative function on $[0, T], T>1$, such that

$$
\phi(t)^{1+r} \leq \omega_{0}(\phi(t)-\phi(t+1)) \text { on }[0, T],
$$

where $\omega_{0}$ is a positive constant and $r$ is a nonnegative constant. Then we have

(i) if $r>0$, then

$$
\phi(t) \leq\left(\phi(0)^{-r}+\omega_{0}^{-1} r[t-1]^{+}\right)^{-\frac{1}{r}} \text { on }[0, T]
$$

(ii) if $r=0$, then

$$
\phi(t) \leq \phi(0) e^{-\omega_{1}[t-1]^{+}} \text {on }[0, T]
$$

where $\omega_{1}=\ln \left(\frac{\omega_{0}}{\omega_{0}-1}\right)$, here $\omega_{0}>1$.

Now, we state the global existence and energy decay results.

Theorem 5.1. Let $u$ be the unique local weak solution to problem (1.1)-(1.2). Suppose that $m \geq 2, p>2$ and $-\Lambda_{1}<a_{1} \leq a \leq a_{2}$ hold. The initial data $\left(u_{0}, u_{1}\right) \in H_{*}^{2}(\Omega) \times L^{2}(\Omega)$ are given. Then $u$ is a global solution,

$$
\lim _{t \rightarrow \infty}\left(\|u(t)\|_{H_{*}^{2}}+\left\|u_{t}(t)\right\|_{2}\right)=0
$$

and

$$
E(t) \leq\left(E(0)^{-\frac{m-2}{2}}+\frac{(m-2) \tau}{2}[t-1]^{+}\right)^{-\frac{2}{m-2}}
$$

if and only if there exists a real number $t_{0} \in\left[0, T_{\max }\right)$ such that

$$
u\left(t_{0}\right) \in W \text { and } E\left(t_{0}\right)<D \text {, }
$$

where $\tau$ is given in (5.18).

Proof. (Sufficiency). Assume that there exists a real number $t_{0} \in\left[0, T_{\max }\right)$ such that

$$
u\left(t_{0}\right) \in W \text { and } E\left(t_{0}\right)<D \text {. }
$$

From Lemma 5.1, we find that $D=\frac{p-2}{2 p} A_{1}^{\frac{p}{p-2}} S_{p}^{-\frac{2 p}{p-2}} \leq d$. Then as the author did in [39, Theorem 3.3], we can get $u(t) \in W$ for all $t \in\left[0, T_{\max }\right)$. So

$$
I(u(t))=\|u(t)\|_{H_{*}^{2}}^{2}+(a u(t), u(t))_{2}-\|u(t)\|_{p}^{p}>0,
$$

which means

$$
\frac{1}{2}\|u(t)\|_{H_{*}^{2}}^{2}+\frac{1}{2}(a u(t), u(t))_{2}-\frac{1}{2}\|u(t)\|_{p}^{p}>0 .
$$

Due to the fact that $p>2$, we have

$$
J(u(t))=\frac{1}{2}\|u(t)\|_{H_{*}^{2}}^{2}+\frac{1}{2}(a u(t), u(t))_{2}-\frac{1}{p}\|u(t)\|_{p}^{p}>0 .
$$

Thus,

For all $t \in\left[0, T_{\max }\right)$,

$$
E(t)=\frac{1}{2}\left\|u_{t}(t)\right\|_{2}^{2}+J(u(t))>0, t \in\left[0, T_{\max }\right) .
$$

$$
\begin{aligned}
J(u(t)) & =\frac{p-2}{2 p}\left(\|u(t)\|_{H_{*}^{2}}^{2}+(a u(t), u(t))_{2}\right)+\frac{I(u(t))}{p} \\
& \geq \frac{p-2}{2 p}\left(\|u(t)\|_{H_{*}^{2}}^{2}+(a u(t), u(t))_{2}\right) .
\end{aligned}
$$

By (2.9), we have

$$
\frac{1}{2}\left\|u_{t}(t)\right\|_{2}^{2}+J(u(t))+\int_{0}^{t}\left\|u_{\tau}(\tau)\right\|_{m}^{m} d \tau=E(0)<d .
$$


Hence,

$$
\frac{1}{2}\left\|u_{t}(t)\right\|_{2}^{2}+\frac{p-2}{2 p}\left(\|u(t)\|_{H_{*}^{2}}^{2}+(a u(t), u(t))_{2}\right)+\int_{0}^{t}\left\|u_{\tau}(\tau)\right\|_{m}^{m} d \tau \leq C .
$$

Let $\phi \in C_{0}^{\infty}(0, T)$, then by equation (1.1) we have

$$
\begin{aligned}
& -\int_{0}^{T}\left(u_{\tau}(\tau), \omega\right)_{2} \phi^{\prime}(\tau) d \tau \\
= & -\int_{0}^{T}(u(\tau), \omega)_{H_{*}^{2}(\Omega)} \phi(\tau) d \tau-\int_{0}^{T}(a u(\tau), \omega)_{2} \phi(\tau) d \tau-\int_{0}^{T}\left(\left|u_{\tau}(\tau)\right|^{m-2} u_{\tau}(\tau), \omega\right)_{2} \phi(\tau) d \tau \\
& +\int_{0}^{T}\left(|u(\tau)|^{p-2} u(\tau), \omega\right)_{2} \phi(\tau) d \tau
\end{aligned}
$$

for all $\omega \in H_{*}^{2}(\Omega)$. This means $u_{t t} \in C^{0}([0, T], \mathcal{H}(\Omega))$. From above estimations, we have $T_{\max }=\infty$ by the continuous principle.

We now turn to the proof of energy decay result.

By (2.9), we have

$$
E^{\prime}(t)=-\int_{\Omega}\left|u_{t}\right|^{m} d x d y
$$

By integrating (5.3) over $[t, t+1], t>0$, we have

$$
E(t)-E(t+1) \equiv D(t)^{m},
$$

where

$$
D(t)^{m}=\int_{t}^{t+1}\left\|u_{\tau}\right\|_{m}^{m} d \tau
$$

By virtue of (5.5) and the continuous imbedding of $L^{m}(\Omega)$ into $L^{2}(\Omega)$, we observe that

$$
\int_{t}^{t+1} \int_{\Omega}\left|u_{\tau}\right|^{2} d x d y d \tau \leq C \int_{t}^{t+1}\left\|u_{\tau}(\tau)\right\|_{m}^{2} d \tau=C D(t)^{2} .
$$

Hence, from (5.6), there exist $t_{1} \in\left[t, t+\frac{1}{4}\right]$ and $t_{2} \in\left[t+\frac{3}{4}, t+1\right]$ such that

$$
\left\|u_{t}\left(t_{i}\right)\right\|_{2}^{2} \leq 4 C D(t)^{2}, i=1,2 \text {. }
$$

Multiplying equation (1.1) by $u$ and integrating it over $\Omega \times\left[t_{1}, t_{2}\right]$, we get

$$
\int_{t_{1}}^{t_{2}}\left\langle u_{\tau \tau}, u\right\rangle d \tau+\int_{t_{1}}^{t_{2}} \int_{\Omega} \triangle^{2} u u d x d y d \tau+\int_{t_{1}}^{t_{2}}(a u, u)_{2} d \tau+\int_{t_{1}}^{t_{2}} \int_{\Omega}\left|u_{\tau}\right|^{m-1} u d x d y d \tau=\int_{t_{1}}^{t_{2}} \int_{\Omega}|u|^{p} d x d y d \tau
$$

Using Lemma 3.1, we have

$$
\int_{t_{1}}^{t_{2}} I(\tau) d \tau=-\int_{t_{1}}^{t_{2}}\left\langle u_{\tau \tau}, u\right\rangle d \tau-\int_{t_{1}}^{t_{2}} \int_{\Omega}\left|u_{\tau}\right|^{m-2} u_{\tau} u d x d y d \tau .
$$

Integrating by parts on the first term of the right hand side of (5.8), we obtain

$$
\int_{t_{1}}^{t_{2}} I(\tau) d \tau \leq \sum_{i=1}^{2}\left\|u_{t}\left(t_{i}\right)\right\|_{2}\left\|u\left(t_{i}\right)\right\|_{2}+\int_{t_{1}}^{t_{2}}\left\|u_{\tau}\right\|_{2}^{2} d \tau-\int_{t_{1}}^{t_{2}} \int_{\Omega}\left|u_{\tau}\right|^{m-2} u_{\tau} u d x d y d \tau .
$$


By Hölder inequality and Lemmas 2.1 and 2.2, we have

$$
\begin{aligned}
\left.\left|\int_{t_{1}}^{t_{2}} \int_{\Omega}\right| u_{\tau}\right|^{m-2} u_{\tau} u d x d y d \tau \mid & \leq \int_{t_{1}}^{t_{2}}\|u\|_{m}\left\|u_{\tau}\right\|_{m}^{m-1} d \tau \\
& \leq S_{m} \int_{t_{1}}^{t_{2}}\|u\|_{H_{*}^{2}}\left\|u_{\tau}\right\|_{m}^{m-1} d \tau \\
& \leq S_{m}\left(\frac{2 p}{(p-2) A_{1}}\right)^{\frac{1}{2}} \sup _{t_{1} \leq s \leq t_{2}} E(s)^{\frac{1}{2}} \int_{t_{1}}^{t_{2}}\left\|u_{\tau}\right\|_{m}^{m-1} d \tau \\
& \leq S_{m}\left(\frac{2 p}{(p-2) A_{1}}\right)^{\frac{1}{2}} D(t)^{m-1} \sup _{t_{1} \leq s \leq t_{2}} E(s)^{\frac{1}{2}} .
\end{aligned}
$$

Using (5.7) and Lemma 2.1, we also have

$$
\left\|u_{t}\left(t_{i}\right)\right\|_{2}\left\|u\left(t_{i}\right)\right\|_{2} \leq\left\|u_{t}\left(t_{i}\right)\right\|_{2} S_{m}\left\|u\left(t_{i}\right)\right\|_{H_{*}^{2}} \leq C D(t) \sup _{t_{1} \leq s \leq t_{2}} E(s)^{\frac{1}{2}} .
$$

Combining (5.6), (5.10) and (5.11), we obtain from (5.9) that

$$
\int_{t_{1}}^{t_{2}} I(\tau) d \tau \leq 2 C D(t) \sup _{t_{1} \leq s \leq t_{2}} E(s)^{\frac{1}{2}}+C D(t)^{2}+S_{m}\left(\frac{2 p}{(p-2) A_{1}}\right)^{\frac{1}{2}} D(t)^{m-1} \sup _{t_{1} \leq s \leq t_{2}} E(s)^{\frac{1}{2}} .
$$

Using (5.1) and Lemma 2.1, 2.2, we have

$$
\begin{aligned}
\|u(t)\|_{p}^{p} & \leq S_{p}^{p}\|u(t)\|_{H_{*}^{2}}^{p} \leq S_{p}^{p} A_{1}^{-\frac{p}{2}}\left(\|u(t)\|_{H_{*}^{2}}^{2}+(a u(t), u(t))_{2}\right)^{\frac{p}{2}} \\
& =S_{p}^{p} A_{1}^{-\frac{p}{2}}\left(\|u(t)\|_{H_{*}^{2}}^{2}+(a u(t), u(t))_{2}\right)^{\frac{p-2}{2}}\left(\|u(t)\|_{H_{*}^{2}}^{2}+(a u(t), u(t))_{2}\right) \\
& \leq S_{p}^{p} A_{1}^{-\frac{p}{2}}\left(\frac{2 p}{p-2} J(u(t))\right)^{\frac{p-2}{2}}\left(\|u(t)\|_{H_{*}^{2}}^{2}+(a u(t), u(t))_{2}\right) \\
& \leq S_{p}^{p} A_{1}^{-\frac{p}{2}}\left(\frac{2 p}{p-2}\right)^{\frac{p-2}{2}} E(0)^{\frac{p-2}{2}}\left(\|u(t)\|_{H_{*}^{2}}^{2}+(a u(t), u(t))_{2}\right),
\end{aligned}
$$

which implies that

$$
\gamma\left(\|u(t)\|_{H_{*}^{2}}^{2}+(a u(t), u(t))_{2}\right) \leq I(u(t))
$$

where $\gamma=1-S_{p}^{p} A_{1}^{-\frac{p}{2}}\left(\frac{2 p}{p-2}\right)^{\frac{p-2}{2}} E(0)^{\frac{p-2}{2}}>0$. We also find that

$$
\|u(t)\|_{p}^{p} \leq S_{p}^{p} A_{1}^{-\frac{p}{2}} A_{2}\left(\frac{2 p}{p-2}\right)^{\frac{p-2}{2}} E(0)^{\frac{p-2}{2}}\|u(t)\|_{H_{*}^{2}}^{2}
$$

and

So

$$
\gamma A_{1}\|u(t)\|_{H_{*}^{2}}^{2} \leq I(u(t)) .
$$

$$
\begin{aligned}
\|u(t)\|_{p}^{p} & \leq S_{p}^{p} A_{1}^{-\frac{p}{2}} A_{2}\left(\frac{2 p}{p-2}\right)^{\frac{p-2}{2}} E(0)^{\frac{p-2}{2}}\|u(t)\|_{H_{*}^{2}}^{2} \\
& \leq \frac{S_{p}^{p} A_{1}^{-\frac{p}{2}} A_{2}\left(\frac{2 p}{p-2}\right)^{\frac{p-2}{2}} E(0)^{\frac{p-2}{2}}}{\gamma A_{1}} I(u(t)) \\
& =C_{1} I(u(t)) .
\end{aligned}
$$


Here, we have used the fact that $A_{1}>0$ because $-\Lambda_{1}<a_{1} \leq a \leq a_{2}$ and $\Lambda_{1}>0$. Thus,

$$
\begin{aligned}
E(t) & =\frac{1}{2}\|u(t)\|_{H_{*}^{2}}^{2}+\frac{1}{2}\left\|u_{t}(t)\right\|_{2}^{2}+\frac{1}{2}(a u(t), u(t))_{2}-\frac{1}{p}\|u(t)\|_{p}^{p} \\
& =\frac{1}{2}\left\|u_{t}(t)\right\|_{2}^{2}+\frac{1}{2} I(u(t))+\frac{p-2}{2 p}\|u(t)\|_{p}^{p} \\
& \leq \frac{1}{2}\left\|u_{t}(t)\right\|_{2}^{2}+\frac{1}{2} I(u(t))+\frac{p-2}{2 p} C_{1} I(u(t)) \\
& \leq \frac{1}{2}\left\|u_{t}(t)\right\|_{2}^{2}+C_{2} I(u(t)) .
\end{aligned}
$$

By integrating (5.15) over $\left(t_{1}, t_{2}\right)$, we obtain

$$
\int_{t_{1}}^{t_{2}} E(\tau) d \tau \leq \frac{1}{2} \int_{t_{1}}^{t_{2}}\left\|u_{\tau}(\tau)\right\|_{2}^{2} d \tau+C_{2} \int_{t_{1}}^{t_{2}} I(\tau) d \tau .
$$

Hence, by (5.6) and (5.12), we have

$$
\begin{aligned}
& \int_{t_{1}}^{t_{2}} E(\tau) d \tau \\
&(5.16) \leq \frac{1}{2} C D(t)^{2}+C_{2}\left\{2 C D(t) \sup _{t_{1} \leq s \leq t_{2}} E(s)^{\frac{1}{2}}+C D(t)^{2}+S_{m}\left(\frac{2 p}{(p-2) A_{1}}\right)^{\frac{1}{2}} D(t)^{m-1} \sup _{t_{1} \leq s \leq t_{2}} E(s)^{\frac{1}{2}}\right\} .
\end{aligned}
$$

Moreover, multiplying equation (1.1) by $u_{t}$ and then integrating it over $\left[t, t_{2}\right] \times \Omega$, we get

$$
\begin{aligned}
& \int_{t}^{t_{2}}\left\langle u_{\tau \tau}, u_{\tau}\right\rangle d \tau+\int_{t}^{t_{2}} \int_{\Omega} \Delta^{2} u u_{\tau} d x d y d \tau+\int_{t}^{t_{2}}\left(a u, u_{\tau}\right) d \tau+\int_{t}^{t_{2}} \int_{\Omega}\left|u_{\tau}\right|^{m} d x d y d \tau \\
= & \int_{t}^{t_{2}} \int_{\Omega}|u|^{p-1} u_{\tau} d x d y d \tau
\end{aligned}
$$

which means

$$
\begin{aligned}
& \frac{1}{2}\left\|u_{t}\left(t_{2}\right)\right\|_{2}^{2}-\frac{1}{2}\left\|u_{t}(t)\right\|_{2}^{2}+\frac{1}{2}\left\|u_{t}\left(t_{2}\right)\right\|_{H_{*}^{2}}^{2}-\frac{1}{2}\left\|u_{t}(t)\right\|_{H_{*}^{2}}^{2}+\frac{1}{2}\left(a u\left(t_{2}\right), u\left(t_{2}\right)\right)-\frac{1}{2}(a u(t), u(t)) \\
& +\frac{1}{p}\|u(t)\|_{p}^{p}-\frac{1}{p}\left\|u\left(t_{2}\right)\right\|_{p}^{p} \\
= & -\int_{t}^{t_{2}}\left\|u_{\tau}(\tau)\right\|_{m}^{m} d \tau .
\end{aligned}
$$

That is

$$
E(t)=E\left(t_{2}\right)+\int_{t}^{t_{2}}\left\|u_{\tau}(\tau)\right\|_{m}^{m} d \tau
$$

Since $t_{2}-t_{1} \geq \frac{1}{2}$, it follows that

$$
E\left(t_{2}\right) \leq 2 \int_{t_{1}}^{t_{2}} E(\tau) d \tau
$$

Then, thanks to (5.4), we arrive at

$$
E(t)=E(t+1)+D(t)^{m} \leq E\left(t_{2}\right)+D(t)^{m} \leq 2 \int_{t_{1}}^{t_{2}} E(\tau) d \tau+D(t)^{m} .
$$


Thus, by using (5.16), we see that

$$
\begin{aligned}
E(t) \leq & 2 C_{2}\left\{2 C D(t) \sup _{t_{1} \leq s \leq t_{2}} E(s)^{\frac{1}{2}}+C D(t)^{2}+S_{m}\left(\frac{2 p}{(p-2) A_{1}}\right)^{\frac{1}{2}} D(t)^{m-1} \sup _{t_{1} \leq s \leq t_{2}} E(s)^{\frac{1}{2}}\right\} \\
& +C D(t)^{2}+D(t)^{m} \\
\leq & \left(C+2 C C_{2}\right) D(t)^{2}+2 C_{2}\left[2 C D(t)+S_{m}\left(\frac{2 p}{(p-2) A_{1}}\right)^{\frac{1}{2}} D(t)^{m-1}\right] \sup _{t_{1} \leq s \leq t_{2}} E(s)^{\frac{1}{2}}+D(t)^{m} \\
\leq & \left(C+2 C C_{2}\right) D(t)^{2}+2 C_{2}\left[2 C D(t)+S_{m}\left(\frac{2 p}{(p-2) A_{1}}\right)^{\frac{1}{2}} D(t)^{m-1}\right] E(t)^{\frac{1}{2}}+D(t)^{m}, t \geq 0 .
\end{aligned}
$$

Using Young's inequality, we deduce

$$
E(t) \leq C_{3}\left[D(t)^{2}+D(t)^{m}+D(t)^{2(m-1)}\right]
$$

where $C_{3}$ is some positive constant. From (5.4) and (5.17), we get

$$
E(t) \leq C_{3}\left[1+D(t)^{m-2}+D(t)^{2 m-4}\right] D(t)^{2} \leq C_{3}\left[1+E(0)^{\frac{m-2}{m}}+E(0)^{\frac{2 m-4}{m}}\right] D(t)^{2} .
$$

This implies that

$$
E(t)^{\frac{m}{2}} \leq\left(C_{4}(E(0))\right)^{\frac{m}{2}} D(t)^{m}
$$

where $C_{4}(E(0))=C_{3}\left[1+E(0)^{\frac{m-2}{m}}+E(0)^{\frac{2 m-4}{m}}\right]$. Note that $\lim _{E(0) \rightarrow 0} C_{4}(E(0))=C_{3}>0$. Hence, by applying Lemma 5.2, we have

$$
E(t) \leq\left(E(0)^{-\frac{m-2}{2}}+\frac{(m-2) \tau}{2}[t-1]^{+}\right)^{-\frac{2}{m-2}} \text { on }[0, \infty),
$$

where $\tau=\left(C_{4}(E(0))\right)^{-\frac{m}{2}}$. Consequently, by (5.1) we immediately have

$$
\lim _{t \rightarrow \infty}\left(\|u(t)\|_{H_{*}^{2}}^{2}+(a u(t), u(t))_{2}+\left\|u_{t}(t)\right\|_{2}^{2}\right)=0,
$$

which combining with Lemma 2.2 tells us that

$$
\lim _{t \rightarrow \infty}\left(\|u(t)\|_{H_{*}^{2}}+\left\|u_{t}(t)\right\|_{2}\right)=0 .
$$

(Necessity). If $T_{\max }=\infty, \lim _{t \rightarrow \infty}\left(\|u(t)\|_{H_{*}^{2}}+\left\|u_{t}(t)\right\|_{2}\right)=0$ and

$$
E(t) \leq\left(E(0)^{-\frac{m-2}{2}}+\frac{(m-2) \tau}{2}[t-1]^{+}\right)^{-\frac{2}{m-2}}
$$

then by Lemma 2.1, 2.2, we have

$$
\lim _{t \rightarrow \infty}\|u(t)\|_{p}=0 \text { and } \lim _{t \rightarrow \infty}\left(\|u(t)\|_{H_{*}^{2}}+(a u(t), u(t))_{2}+\left\|u_{t}(t)\right\|_{2}\right)=0
$$

which imply that

$$
\lim _{t \rightarrow \infty} E(t)=0
$$

By Lemma 2.4 and the above mentioned results, there must be $t_{0}>0$ such that $E\left(t_{0}\right)<D$ with $u\left(t_{0}\right) \in W$. 


\section{BlOW UP}

In this section, we prove the blow-up properties of solutions.

Theorem 6.1. Let $u$ be the unique local weak solution to problem (1.1)-(1.2). Suppose that $p>m \geq 2$ and $-\Lambda_{1}<a_{1} \leq a \leq a_{2}$ hold. The initial data $\left(u_{0}, u_{1}\right) \in H_{*}^{2}(\Omega) \times L^{2}(\Omega)$ are given. If there exists a real number $t_{0} \in\left[0, T_{\max }\right)$ such that

$$
u\left(t_{0}\right) \in U \text { and } E\left(t_{0}\right)<D
$$

then $u$ blows up, that is,

$$
T_{\max }<\infty
$$

Proof. Assume that there exists a real number $t_{0} \in\left[0, T_{\max }\right)$ such that

$$
u\left(t_{0}\right) \in U \text { and } E\left(t_{0}\right)<D .
$$

Without loss of generality, we may suppose that $t_{0}=0$, then

$$
u(t) \in U \text { and } E(t)<D, \quad \text { for every } t \in\left[0, T_{\max }\right) .
$$

Indeed, (2.9) entails that

$$
E(t) \leq E(0)<D, \quad \text { for all } t>0 .
$$

Then suppose that there exists $\bar{t}>0$ such that $u(\bar{t}) \in N$, but by (2.7),

$$
d \leq J(u(\bar{t})) \leq E(\bar{t})<D,
$$

which is a contradiction to (6.1), and therefore $u(t) \in U$ for all $t \in\left[0, T_{\max }\right)$.

We divide our proof into two cases:

Case 1. Assume that $u(t) \in U$ and $0 \leq E(t)<D$, for every $t \in\left[0, T_{\max }\right)$.

From (2.8), we know that

$$
\begin{aligned}
E(t) & =\frac{1}{2}\|u(t)\|_{H_{*}^{2}}^{2}+\frac{1}{2}\left\|u_{t}(t)\right\|_{2}^{2}+\frac{1}{2}(a u(t), u(t))_{2}-\frac{1}{p}\|u(t)\|_{p}^{p} \\
& \geq \frac{A_{1}}{2}\|u(t)\|_{H_{*}^{2}}^{2}+\frac{1}{2}\left\|u_{t}(t)\right\|_{2}^{2}-\frac{1}{p} S_{p}^{p}\|u(t)\|_{H_{*}^{2}}^{p} .
\end{aligned}
$$

We define a functional $\varepsilon(t)$ as follows:

$$
\varepsilon(t):=\varepsilon(u(t))=\frac{A_{1}}{2}\|u(t)\|_{H_{*}^{2}}^{2}+\frac{1}{2}\left\|u_{t}(t)\right\|_{2}^{2} .
$$

Thus,

So,

$$
\|u(t)\|_{H_{*}^{2}} \leq\left(\frac{2}{A_{1}} \varepsilon(t)\right)^{\frac{1}{2}}
$$

$$
E(t) \geq \varepsilon(t)-\frac{1}{p} S_{p}^{p}\left(\frac{2}{A_{1}} \varepsilon(t)\right)^{\frac{p}{2}}=\varepsilon(t)-\frac{1}{p}\left(\frac{2}{A_{1}} S_{p}^{2} \varepsilon(t)\right)^{\frac{p}{2}}
$$

for $t \in\left[0, T_{\max }\right)$.

Let us define the function $F: R^{+} \rightarrow R$ by

$$
F(y)=y-\frac{1}{p}\left(\frac{2}{A_{1}} S_{p}^{2} y\right)^{\frac{p}{2}}
$$

By some simple calculations, we find that $F(y)$ obtains its maximum in $[0, \infty)$ at $y=y_{0}$, where

$$
y_{0}=\frac{1}{2} A_{1}^{\frac{p}{p-2}} S_{p}^{-\frac{2 p}{p-2}}
$$

and the maximum value of $F(y)$ is

$$
F\left(y_{0}\right)=\frac{p-2}{2 p} A_{1}^{\frac{p}{p-2}} S_{p}^{-\frac{2 p}{p-2}}=D .
$$


Then inequality (6.2) takes the concise form

$$
E(t) \geq F(\varepsilon(t)), \quad \text { for } t \in\left[0, T_{\max }\right) .
$$

Since the continuous function $F(y)$ attains its maximum value at $y=y_{0}$, it is monotone decreasing when $y>y_{0}$. Due to the fact that $E(0)<D=F\left(y_{0}\right)$, there exists a unique number $y_{1}$ such that

$$
F\left(y_{1}\right)=E(0), \quad \text { with } y_{1}>y_{0}>0 .
$$

Therefore, we have

$$
D>F\left(y_{1}\right)=E(0) \geq E(t) \geq F(\varepsilon(t)), \quad \text { for } t \in\left[0, T_{\max }\right) .
$$

We aim to show that $\varepsilon(0)>y_{0}$.

Indeed, we have proved that $u(t) \in U$ for all $t \in\left[0, T_{\max }\right)$, then $I\left(u_{0}\right)<0$, which means

$$
\left\|u_{0}\right\|_{H_{*}^{2}}^{2}+\left(a u_{0}, u_{0}\right)_{2}-\left\|u_{0}\right\|_{p}^{p}<0 .
$$

By using Lemma 2.1 and 2.2, we have

$$
A_{1}\left\|u_{0}\right\|_{H_{*}^{2}}^{2}<S_{p}^{p}\left\|u_{0}\right\|_{H_{*}^{2}}^{p}
$$

that is

$$
\left\|u_{0}\right\|_{H_{*}^{2}}>\left(\frac{A_{1}}{S_{p}^{p}}\right)^{\frac{1}{p-2}}
$$

Since $\varepsilon(t)=\frac{A_{1}}{2}\|u(t)\|_{H_{*}^{2}}^{2}+\frac{1}{2}\left\|u_{t}(t)\right\|_{2}^{2} \geq \frac{A_{1}}{2}\|u(t)\|_{H_{*}^{2}}^{2}$, we have

$$
\varepsilon(0) \geq \frac{A_{1}}{2}\left\|u_{0}\right\|_{H_{*}^{2}}^{2}>\frac{A_{1}}{2}\left(\frac{A_{1}}{S_{p}^{p}}\right)^{\frac{2}{p-2}}=\frac{1}{2} A_{1}^{\frac{p}{p-2}} S_{p}^{-\frac{2 p}{p-2}}=y_{0} .
$$

Since $F(y)$ is continuous and decreasing when $y>y_{0}$ and $\varepsilon(t)$ is also continuous, then by using (6.6), it follows from (6.5) that

$$
\varepsilon(t) \geq y_{1}, \quad \text { for } t \in\left[0, T_{\max }\right) \text {. }
$$

Consequently,

$$
\begin{aligned}
\frac{1}{p}\|u(t)\|_{p}^{p} & =\frac{1}{2}\|u(t)\|_{H_{*}^{2}}^{2}+\frac{1}{2}\left\|u_{t}(t)\right\|_{2}^{2}+\frac{1}{2}(a u(t), u(t))_{2}-E(t) \\
& \geq \frac{A_{1}}{2}\|u(t)\|_{H_{*}^{2}}^{2}+\frac{1}{2}\left\|u_{t}(t)\right\|_{2}^{2}-E(t) \\
& =\varepsilon(t)-E(t) \geq y_{1}-F\left(y_{1}\right)=\frac{1}{p}\left(\frac{2}{A_{1}} S_{p}^{2} y_{1}\right)^{\frac{p}{2}},
\end{aligned}
$$

which can be reduced to

$$
\|u(t)\|_{p}^{p} \geq\left(\frac{2}{A_{1}} S_{p}^{2} y_{1}\right)^{\frac{p}{2}}, \quad \text { for } t \in\left[0, T_{\max }\right) .
$$

Now we set $G(t)=D-E(t)>0$ and $N(t)=\frac{1}{2}\|u(t)\|_{2}^{2}$ for $t \in\left[0, T_{\max }\right)$. We aim to show that

$$
Y(t)=G^{1-\alpha}(t)+\epsilon N^{\prime}(t)
$$

blows up in finite time, for some $\alpha \in(0,1)$ and $\epsilon>0$, which will be selected later. By differentiating both sides of $(6.9)$, one has

(6.10) $Y^{\prime}(t)=(1-\alpha) G^{-\alpha}(t) G^{\prime}(t)+\epsilon\left[\left\|u_{t}(t)\right\|_{2}^{2}-\|u(t)\|_{H_{*}^{2}}^{2}-(a u(t), u(t))_{2}-\int_{\Omega}\left|u_{t}\right|^{m-1} u d x+\|u(t)\|_{p}^{p}\right]$. 
By using (6.7) and the fact that $D=F\left(y_{0}\right)=\frac{p-2}{2 p} A_{1}^{\frac{p}{p-2}} S_{p}^{-\frac{2 p}{p-2}}$, we obtain

$$
\begin{aligned}
G(t)=D-E(t) & \leq D-\varepsilon(t)+\frac{1}{p}\|u(t)\|_{p}^{p} \\
& \leq y_{0}-\frac{1}{p}\left(\frac{2}{A_{1}} S_{p}^{2} y_{0}\right)^{\frac{p}{2}}-y_{1}+\frac{1}{p}\|u(t)\|_{p}^{p} \\
& =\left(y_{0}-y_{1}\right)-\frac{1}{p}\left(\frac{2}{A_{1}} S_{p}^{2} y_{0}\right)^{\frac{p}{2}}+\frac{1}{p}\|u(t)\|_{p}^{p} \\
& <-\frac{1}{p}\left(\frac{2}{A_{1}} S_{p}^{2} y_{0}\right)^{\frac{p}{2}}+\frac{1}{p}\|u(t)\|_{p}^{p}
\end{aligned}
$$

since $y_{1}>y_{0}$. The last inequality can be expressed as

$$
\|u(t)\|_{p}^{p}>p G(t)+\left(\frac{2}{A_{1}} S_{p}^{2} y_{0}\right)^{\frac{p}{2}} .
$$

Also, since $G(t)=D-E(t)$, then by the energy identity (2.9), we have

$$
G^{\prime}(t)=-E^{\prime}(t)=\left\|u_{t}(t)\right\|_{m}^{m}
$$

which shows that $G(t)$ is nondecreasing for $t \in\left[0, T_{\max }\right)$. By applying Hö lder's and Young's inequalities, and using the assumption that $p>m$, we obtain

$$
\begin{aligned}
\left.\left|\int_{\Omega}\right| u_{t}\right|^{m-1} u d x \mid & \leq\|u(t)\|_{m}\left\|u_{t}(t)\right\|_{m}^{m-1} \\
& \leq C\|u(t)\|_{p}\left\|u_{t}(t)\right\|_{m}^{m-1} \\
& =C\|u(t)\|_{p}^{1-\frac{p}{m}}\left(\|u(t)\|_{p}^{\frac{p}{m}}\left\|u_{t}(t)\right\|_{m}^{m-1}\right) \\
& \leq C G(t)^{\frac{1}{p}-\frac{1}{m}}\left(\|u(t)\|_{p}^{\frac{p}{m}}\left\|u_{t}(t)\right\|_{m}^{m-1}\right) \\
& \leq \lambda G(t)^{\frac{1}{p}-\frac{1}{m}}\|u(t)\|_{p}^{p}+C_{\lambda} G(t)^{\frac{1}{p}-\frac{1}{m}}\left\|u_{t}(t)\right\|_{m}^{m} \\
& \leq \lambda G(0)^{\frac{1}{p}-\frac{1}{m}}\|u(t)\|_{p}^{p}+C_{\lambda} G(0)^{\frac{1}{p}-\frac{1}{m}+\alpha} G(t)^{-\alpha} G^{\prime}(t),
\end{aligned}
$$

where we choose $0<\alpha<\frac{1}{m}-\frac{1}{p}$, and $\lambda$ is a positive constant which will be selected later. Thus,

$$
\begin{aligned}
Y^{\prime}(t) \geq & {\left[1-\alpha-\epsilon C_{\lambda} G(0)^{\frac{1}{p}-\frac{1}{m}+\alpha}\right] G(t)^{-\alpha} G^{\prime}(t) } \\
& +\epsilon\left[\left\|u_{t}(t)\right\|_{2}^{2}-\|u(t)\|_{H_{*}^{2}}^{2}-(a u(t), u(t))_{2}+\left(1-\lambda G(0)^{\frac{1}{p}-\frac{1}{m}}\right)\|u(t)\|_{p}^{p}\right] .
\end{aligned}
$$

Since

$$
\|u(t)\|_{H_{*}^{2}}^{2}+(a u(t), u(t))_{2}=2 E(t)+\frac{2}{p}\|u(t)\|_{p}^{p}-\left\|u_{t}(t)\right\|_{2}^{2}
$$

we have

$$
\begin{aligned}
Y^{\prime}(t) \geq & {\left[1-\alpha-\epsilon C_{\lambda} G(0)^{\frac{1}{p}-\frac{1}{m}+\alpha}\right] G(t)^{-\alpha} G^{\prime}(t) } \\
& +\epsilon\left[2\left\|u_{t}(t)\right\|_{2}^{2}+\left(1-\lambda G(0)^{\frac{1}{p}-\frac{1}{m}}-\frac{2}{p}\right)\|u(t)\|_{p}^{p}-2 E(t)\right],
\end{aligned}
$$

for $t \in\left[0, T_{\max }\right)$.

Next, we aim to show that

$$
\left(1-\frac{2}{p}\right)\|u(t)\|_{p}^{p}-2 E(t)>c\|u(t)\|_{p}^{p}, \quad \text { for all } t \in\left[0, T_{\max }\right)
$$


for some $c>0$. For the sake of convenience, we put

$$
C_{0}=\left(\frac{2}{A_{1}} S_{p}^{2} y_{1}\right)^{\frac{p}{2}}
$$

Then we have

$$
0 \leq E(t) \leq E(0)=F\left(y_{1}\right)=y_{1}-\frac{1}{p}\left(\frac{2}{A_{1}} S_{p}^{2} y_{1}\right)^{\frac{p}{2}}=y_{1}-\frac{1}{p} C_{0},
$$

for $t \in\left[0, T_{\max }\right)$.

Now we split the term $\left(1-\frac{2}{p}\right)\|u(t)\|_{p}^{p}$ into two positive parts:

$$
\frac{p-2}{p}\|u(t)\|_{p}^{p}=\left(\frac{p-2}{p}-\frac{C_{0}-2 y_{1}}{2 C_{0}}\right)\|u(t)\|_{p}^{p}+\frac{C_{0}-2 y_{1}}{2 C_{0}}\|u(t)\|_{p}^{p} .
$$

The fact that the two terms on the right-hand side of (6.18) are both positive from the following straightforward calculations. Indeed, by $(6.16)$ and the fact that $y_{1}>y_{0}=\frac{1}{2} A_{1}^{\frac{p}{p-2}} S_{p}^{-\frac{2 p}{p-2}}$, we compute

$$
\begin{aligned}
C_{0}-2 y_{1} & =\left[\left(\frac{2}{A_{1}} S_{p}^{2}\right)^{\frac{p}{2}} y_{1}^{\frac{p}{2}-1}-2\right] y_{1} \\
& >\left[\left(\frac{2}{A_{1}} S_{p}^{2}\right)^{\frac{p}{2}} y_{0}^{\frac{p}{2}-1}-2\right] y_{1} \\
& =\left[\left(\frac{2}{A_{1}} S_{p}^{2}\right)^{\frac{p}{2}}\left(\frac{1}{2} A_{1}^{\frac{p}{p-2}} S_{p}^{-\frac{2 p}{p-2}}\right)^{\frac{p-2}{2}}-2\right] y_{1} \\
& =\left[2 A_{1}^{-\frac{p}{2}+\frac{p}{2}} S_{p}^{p-p}-2\right] y_{1}=0,
\end{aligned}
$$

and

$$
\begin{aligned}
\frac{p-2}{p}-\frac{C_{0}-2 y_{1}}{2 C_{0}} & =\frac{2 C_{0}(p-2)-p\left(C_{0}-2 y_{1}\right)}{2 C_{0} p}=\frac{C_{0}(p-2)+2 p y_{1}-2 C_{0}}{2 C_{0} p} \\
& =\frac{C_{0}(p-2)+2\left(p y_{1}-C_{0}\right)}{2 C_{0} p}=\frac{C_{0}(p-2)+p F\left(y_{1}\right)}{2 C_{0} p} \geq 0 .
\end{aligned}
$$

Thus, we can define the positive constant $c$ as

$$
c:=\frac{C_{0}-2 y_{1}}{2 C_{0}}>0
$$

Applying (6.20) and (6.21) along with the fact that $\|u(t)\|_{p}^{p} \geq C_{0}$ from (6.8), we obtain from (6.18) that

$$
\frac{p-2}{p}\|u(t)\|_{p}^{p} \geq\left(\frac{p-2}{p}-\frac{C_{0}-2 y_{1}}{2 C_{0}}\right) C_{0}+c\|u(t)\|_{p}^{p} .
$$

By using (6.17) and (6.22), we calculate

$$
\begin{aligned}
\frac{p-2}{p}\|u(t)\|_{p}^{p}-2 E(t) & \geq\left(\frac{p-2}{p}-\frac{C_{0}-2 y_{1}}{2 C_{0}}\right) C_{0}+c\|u(t)\|_{p}^{p}-2 E(t) \\
& \geq\left(\frac{p-2}{p}-\frac{C_{0}-2 y_{1}}{2 C_{0}}\right) C_{0}-2 y_{1}+\frac{2}{p} C_{0}+c\|u(t)\|_{p}^{p} \\
& =\frac{C_{0}}{2}-y_{1}+c\|u(t)\|_{p}^{p} \\
& \geq c\|u(t)\|_{p}^{p}
\end{aligned}
$$

where the last inequality follows from (6.19). 
Applying (6.23) to (6.15) yields

$$
\begin{aligned}
Y^{\prime}(t)> & {\left[1-\alpha-\epsilon C_{\lambda} G(0)^{\frac{1}{p}-\frac{1}{m}+\alpha}\right] G(t)^{-\alpha} G^{\prime}(t) } \\
& +\epsilon\left[2\left\|u_{t}(t)\right\|_{2}^{2}+\left(c-\lambda G(0)^{\frac{1}{p}-\frac{1}{m}}\right)\|u(t)\|_{p}^{p}\right] .
\end{aligned}
$$

Now, we choose $\lambda>0$ such that $\lambda G(0)^{\frac{1}{p}-\frac{1}{m}}=\frac{c}{2}$ and select $\epsilon>0$ sufficiently small so that $\epsilon C_{\lambda} G(0)^{\frac{1}{p}-\frac{1}{m}+\alpha} \leq$ $1-\alpha$, we obtain from $(6.24)$ that

$$
Y^{\prime}(t) \geq \frac{\epsilon}{2}\left[2\left\|u_{t}(t)\right\|_{2}^{2}+c\|u(t)\|_{p}^{p}\right], \quad \text { for } t \in\left[0, T_{\max }\right) .
$$

Combining the estimates (6.25) and (6.11) yields that

$$
Y^{\prime}(t) \geq \frac{\epsilon}{2}\left[2\left\|u_{t}(t)\right\|_{2}^{2}+\frac{c}{2}\|u(t)\|_{p}^{p}+\frac{c}{2} p G(t)\right]>0
$$

for $t \in\left[0, T_{\max }\right)$, where the last inequality is due to the fact that $G(t)=D-E(t)>0$.

Notice that $Y(0)=G(0)^{1-\alpha}+\epsilon N^{\prime}(0)$, and if $N^{\prime}(0)<0$, then we shall further impose the restriction $0<\epsilon \leq-\frac{G(0)^{1-\alpha}}{2 N^{\prime}(0)}$ so that $Y(0) \geq \frac{1}{2} G(0)^{1-\alpha}$. Since $Y(t)$ is increasing on $\left[0, T_{\max }\right)$ by virtue of $(6.26)$, it follows that

$$
Y(t) \geq Y(0) \geq \frac{1}{2} G(0)^{1-\alpha}>0, \quad \text { for } t \in\left[0, T_{\max }\right) .
$$

Since $Y(t)=G(t)^{1-\alpha}+\epsilon N^{\prime}(t)$, if we let $\epsilon \leq 1$, it follows that

$$
Y(t)^{\frac{1}{1-\alpha}} \leq C\left(G(t)+\left|N^{\prime}(t)\right|^{\frac{1}{1-\alpha}}\right), \quad \text { for } t \in\left[0, T_{\max }\right) .
$$

By the Cauchy-Schwarz and Young's inequalities, we have

$$
\left|N^{\prime}(t)\right|^{\frac{1}{1-\alpha}} \leq\left\|u_{t}(t)\right\|_{2}^{\frac{1}{1-\alpha}}\|u(t)\|_{2}^{\frac{1}{1-\alpha}} \leq C\left\|u_{t}(t)\right\|_{2}^{\frac{1}{1-\alpha}}\|u(t)\|_{p^{\frac{1}{1-\alpha}}}^{11} \leq C\left(\left\|u_{t}(t)\right\|_{2}^{2}+\|u(t)\|_{p}^{\frac{2}{1-2 \alpha}}\right) .
$$

Notice that

$$
\|u(t)\|_{p}^{\frac{2}{1-2 \alpha}}=\left(\|u(t)\|_{p}^{p}\right)^{\frac{2}{(1-2 \alpha) p}}=\left(\|u(t)\|_{p}^{p}\right)^{\frac{2}{(1-2 \alpha) p}-1}\|u(t)\|_{p}^{p} .
$$

Now we impose an extra restriction on $\alpha$ :

$$
0<\alpha<\frac{p-2}{2 p}
$$

then $\sigma:=1-\frac{2}{(1-2 \alpha) p}>0$. By virtue of $(6.11)$ and the fact that $G(t)$ is nondecreasing for $t \in\left[0, T_{\max }\right)$, and letting $0<\epsilon \leq G(0)$, it follows from (6.30) that

$$
\|u(t)\|_{p}^{\frac{2}{1-2 \alpha}}=\left(\|u(t)\|_{p}^{p}\right)^{-\sigma}\|u(t)\|_{p}^{p} \leq C G(t)^{-\sigma}\|u(t)\|_{p}^{p} \leq C G(0)^{-\sigma}\|u(t)\|_{p}^{p} \leq C \epsilon^{-\sigma}\|u(t)\|_{p}^{p} .
$$

By substituting the above inequality into (6.29), one has

$$
\left|N^{\prime}(t)\right|^{\frac{1}{1-\alpha}} \leq C\left(\left\|u_{t}(t)\right\|_{2}^{2}+\epsilon^{-\sigma}\|u(t)\|_{p}^{p}\right), \quad \text { for } t \in\left[0, T_{\max }\right) .
$$

Since $\epsilon \leq 1$ and $\sigma>0$, then

$$
\left|N^{\prime}(t)\right|^{\frac{1}{1-\alpha}} \leq C \epsilon^{-\sigma}\left(\left\|u_{t}(t)\right\|_{2}^{2}+\|u(t)\|_{p}^{p}\right), \quad \text { for } t \in\left[0, T_{\max }\right),
$$

and along with (6.28), it follows that

$$
Y(t)^{\frac{1}{1-\alpha}} \leq C \epsilon^{-\sigma}\left(G(t)+\left\|u_{t}(t)\right\|_{2}^{2}+\|u(t)\|_{p}^{p}\right), \quad \text { for } t \in\left[0, T_{\max }\right) .
$$

By taking into account inequalities (6.26) and (6.32), we see that

$$
Y^{\prime}(t) \geq C(p, E(0)) \epsilon^{1+\sigma} Y(t)^{\frac{1}{1-\alpha}}, \quad \text { for } t \in\left[0, T_{\max }\right),
$$


and since $\frac{1}{1-\alpha}>1$, we conclude that $T_{\max }$ is necessarily finite. More precisely,

$$
T_{\max }<\frac{1-\alpha}{\alpha} \epsilon^{-(1+\sigma)} C(p, E(0)) Y(0)^{-\frac{\alpha}{1-\alpha}} \leq \frac{1-\alpha}{\alpha} \epsilon^{-(1+\sigma)} C(p, E(0)) G(0)^{-\alpha},
$$

where the last inequality comes from (6.27).

Case 2. Assume that $u(t) \in U$ and $E(t)<0$, for every $t \in\left[0, T_{\max }\right)$.

In this case, we put $G(t)=-E(t)$ and $N(t)=\frac{1}{2}\|u(t)\|_{2}^{2}$. We also aim to show that

$$
Y(t)=G(t)^{1-\alpha}+\epsilon N^{\prime}(t)
$$

blows up in finite time, for some $\alpha \in(0,1)$ and $\epsilon>0$, which will be selected later.

We calculate

$$
Y^{\prime}(t)=(1-\alpha) G(t)^{-\alpha} G^{\prime}(t)+\epsilon N^{\prime \prime}(t)
$$

where

$$
N^{\prime \prime}(t)=\left\|u_{t}(t)\right\|_{2}^{2}-\|u(t)\|_{H_{*}^{2}}^{2}-(a u(t), u(t))_{2}-\int_{\Omega}\left|u_{t}\right|^{m-1} u d x+\|u(t)\|_{p}^{p} .
$$

The next step is to find an appropriate lower bound of right-hand side of (6.35). By applying Hölder's and Young's inequalities, and using the assumption that the source is stronger than damping, i.e., $p>m$, we obtain

$$
\left.\left|\int_{\Omega}\right| u_{t}\right|^{m-1} u d x \mid \leq\|u(t)\|_{m}\left\|u_{t}(t)\right\|_{m}^{m-1} \leq C\|u(t)\|_{p}\left\|u_{t}(t)\right\|_{m}^{m-1} .
$$

Since $G(t)=-E(t)$, we have

$$
G^{\prime}(t)=\left\|u_{t}(t)\right\|_{m}^{m} \geq 0
$$

Thus, $G(t)$ is nondecreasing for $t \in\left[0, T_{\max }\right)$. Moreover, by (2.8),

$$
G(t)=-E(t) \leq-\frac{A_{1}}{2}\|u(t)\|_{H_{*}^{2}}^{2}-\frac{1}{2}\left\|u_{t}(t)\right\|_{2}^{2}+\frac{1}{p}\|u(t)\|_{p}^{p} \leq \frac{1}{p}\|u(t)\|_{p}^{p} .
$$

Now, by applying (6.38) to inequality (6.36) and invoking the assumption $p>m$, we deduce

$$
\begin{aligned}
\left.\left|\int_{\Omega}\right| u_{t}\right|^{m-1} u d x \mid & \leq C\|u(t)\|_{p}^{1-\frac{p}{m}}\left(\|u(t)\|_{p}^{\frac{p}{m}}\left\|u_{t}(t)\right\|_{m}^{m-1}\right) \\
& \leq C G(t)^{\frac{1}{p}-\frac{1}{m}}\left(\|u(t)\|_{p}^{\frac{p}{m}}\left\|u_{t}(t)\right\|_{m}^{m-1}\right) \\
& \leq \lambda G(t)^{\frac{1}{p}-\frac{1}{m}}\|u(t)\|_{p}^{p}+C_{\lambda} G(t)^{\frac{1}{p}-\frac{1}{m}}\left\|u_{t}(t)\right\|_{m}^{m}
\end{aligned}
$$

where we have used the Young's inequality and the value of the positive number $\lambda$ will be determined later. By selecting

and using (6.37), we obtain

$$
0<\alpha<\frac{1}{m}-\frac{1}{p}
$$

$$
\begin{aligned}
\left.\left|\int_{\Omega}\right| u_{t}\right|^{m-1} u d x \mid & \leq \lambda G(t)^{\frac{1}{p}-\frac{1}{m}}\|u(t)\|_{p}^{p}+C_{\lambda} G(t)^{\frac{1}{p}-\frac{1}{m}+\alpha} G(t)^{-\alpha}\left\|u_{t}(t)\right\|_{m}^{m} \\
& \leq \lambda G(0)^{\frac{1}{p}-\frac{1}{m}}\|u(t)\|_{p}^{p}+C_{\lambda} G(0)^{\frac{1}{p}-\frac{1}{m}+\alpha} G(t)^{-\alpha} G^{\prime}(t) .
\end{aligned}
$$

By employing (6.40), we obtain from (6.35) that

$$
\begin{aligned}
N^{\prime \prime}(t) \geq & \left\|u_{t}(t)\right\|_{2}^{2}-\|u(t)\|_{H_{*}^{2}}^{2}-(a u(t), u(t))_{2} \\
& +\left(1-\lambda G(0)^{\frac{1}{p}-\frac{1}{m}}\right)\|u(t)\|_{p}^{p}-C_{\lambda} G(0)^{\frac{1}{p}-\frac{1}{m}+\alpha} G(t)^{-\alpha} G^{\prime}(t) .
\end{aligned}
$$

Since

$$
E(t)=\frac{1}{2}\|u(t)\|_{H_{*}^{2}}^{2}+\frac{1}{2}(a u(t), u(t))_{2}+\frac{1}{2}\left\|u_{t}(t)\right\|_{2}^{2}-\frac{1}{p}\|u(t)\|_{p}^{p},
$$


we have

$$
\begin{gathered}
N^{\prime \prime}(t) \geq 2\left\|u_{t}(t)\right\|_{2}^{2}+\left(1-\lambda G(0)^{\frac{1}{p}-\frac{1}{m}}-\frac{2}{p}\right)\|u(t)\|_{p}^{p} \\
-C_{\lambda} G(0)^{\frac{1}{p}-\frac{1}{m}+\alpha} G(t)^{-\alpha} G^{\prime}(t)-2 E(t),
\end{gathered}
$$

for $t \in\left[0, T_{\max }\right)$. We choose $\lambda>0$ such that

$$
\lambda G(0)^{\frac{1}{p}-\frac{1}{m}}=\frac{p-2}{2 p},
$$

then inequality $(6.42)$ can be reduced to

$$
N^{\prime \prime}(t) \geq 2\left\|u_{t}(t)\right\|_{2}^{2}+\frac{p-2}{2 p}\|u(t)\|_{p}^{p}-C_{\lambda} G(0)^{\frac{1}{p}-\frac{1}{m}+\alpha} G(t)^{-\alpha} G^{\prime}(t)+2 G(t),
$$

for all $t \in\left[0, T_{\max }\right)$. Now, since $Y^{\prime}(t)=(1-\alpha) G(t)^{-\alpha} G^{\prime}(t)+\epsilon N^{\prime \prime}(t)$, if we select $\epsilon>0$ small enough so that

$$
\epsilon C_{\lambda} G(0)^{\frac{1}{p}-\frac{1}{m}+\alpha} \leq 1-\alpha,
$$

then one has

$$
Y^{\prime}(t) \geq 2 \epsilon\left\|u_{t}(t)\right\|_{2}^{2}+2 \epsilon G(t)+\frac{p-2}{2 p} \epsilon\|u(t)\|_{p}^{p},
$$

for $t \in\left[0, T_{\max }\right)$.

Recall that $G(0)=-E(0)>0$, and since $G(t)$ is nondecreasing by (6.37), it follows that $G(t)>0$ for $t \in\left[0, T_{\max }\right)$. Thanks to $(6.43)$, we have $Y^{\prime}(t)>0$, i.e., $Y(t)$ is monotone increasing for $t \in\left[0, T_{\max }\right)$. Note that $Y(0)=G(0)^{1-\alpha}+\epsilon N^{\prime}(0)$. In case $N^{\prime}(0)<0$, in order to make sure that $Y(0)>0$, we shall impose an extra restriction on $\epsilon$ :

$$
0<\epsilon \leq-\frac{G(0)^{1-\alpha}}{2 N^{\prime}(0)}
$$

As a result,

$$
Y(t) \geq Y(0) \geq \frac{1}{2} G(0)^{1-\alpha}>0, \quad \text { for all } t \in\left[0, T_{\max }\right) .
$$

Also, by following the estimates (6.28)-(6.32) in Case 1, and by imposing the additional restrictions on $\alpha$ and $\epsilon$, namely, $0<\alpha<\frac{p-2}{2 p}$ and $0<\epsilon \leq \min \{G(0), 1\}$, we obtain

$$
Y(t)^{\frac{1}{1-\alpha}} \leq C \epsilon^{-\sigma}\left(G(t)+\left\|u_{t}(t)\right\|_{2}^{2}+\|u(t)\|_{p}^{p}\right), \quad \text { for } t \in\left[0, T_{\max }\right),
$$

where $\sigma=1-\frac{2}{(1-2 \alpha) p}>0$.

By taking into account inequalities (6.43) and (6.45), we see that

$$
Y^{\prime}(t) \geq \epsilon^{1+\sigma} C(p) Y(t)^{\frac{1}{1-\alpha}}, \quad \text { for } t \in\left[0, T_{\max }\right),
$$

and since $\frac{1}{1-\alpha}>1$, we conclude that $T_{\max }$ is necessarily finite. More precisely,

$$
T_{\max }<\frac{1-\alpha}{\alpha} \epsilon^{-(1+\sigma)} C(p) Y(0)^{-\frac{\alpha}{1-\alpha}} \leq \frac{1-\alpha}{\alpha} \epsilon^{-(1+\alpha)} C(p) G(0)^{-\alpha},
$$

where the last inequality is due to (6.44).

The proof is completed. 
7. LOWER BOUND FOR BLOW-UP TIME

In this section, we present the lower bound of the blow-up time $T_{\max }$.

Theorem 7.1. Under the same assumptions as in Theorem 6.1. If u occurs blow-up at a finite time $T_{\max }$, then

$$
T_{\text {max }} \geq \int_{F(0)}^{\infty} \frac{d y}{p E(0)+y+\frac{p S_{2(p-1)}^{2(p-1)} 2^{2 p-4}}{A_{1}^{p-1}} E(0)^{p-1}+\frac{S_{2(p-1)}^{2(p-1)} 2^{2 p-4}}{A_{1}^{p-1} p^{p-2}} y^{p-1}} .
$$

Proof. First, due to the fact that $-\Lambda_{1}<a_{1} \leq a \leq a_{2}$ and $\Lambda_{1}>0$, we have $A_{1}>0$. Then by Lemma 2.2, we have

$$
\frac{A_{1}}{2}\|u(t)\|_{H_{*}^{2}}^{2}+\frac{1}{2}\left\|u_{t}(t)\right\|_{2}^{2} \leq E(t)+\frac{1}{p}\|u(t)\|_{p}^{p} \leq E(0)+\frac{1}{p}\|u(t)\|_{p}^{p} .
$$

In order to obtain the lower bound of the blow-up time $T_{\max }$, we define the auxiliary functional as follow:

$$
F(t):=F(u(t))=\int_{\Omega}|u|^{p} d x .
$$

It is easy to obtain that $F^{\prime}(t)=p \int_{\Omega}|u|^{p-2} u u_{t} d x$.

Using Cauchy's inequality, we have

$$
F^{\prime}(t)=p \int_{\Omega}|u|^{p-2} u u_{t} d x \leq \frac{p}{2}\left(\int_{\Omega}\left|u_{t}\right|^{2} d x+\int_{\Omega}|u|^{2(p-1)} d x\right) .
$$

By Lemma 2.1 and (7.1), we have

$$
\begin{aligned}
F^{\prime}(t) & \leq \frac{p}{2}\left(\left\|u_{t}(t)\right\|_{2}^{2}+S_{2(p-1)}^{2(p-1)}\|u(t)\|_{H_{*}^{2}}^{2(p-1)}\right) \\
& \leq \frac{p}{2}\left(2 E(0)+\frac{2}{p} F(t)+S_{2(p-1)}^{2(p-1)}\left[\frac{2}{A_{1}}\left(E(0)+\frac{1}{p} F(t)\right)\right]^{p-1}\right) \\
& \leq \frac{p}{2}\left(2 E(0)+\frac{2}{p} F(t)+S_{2(p-1)}^{2(p-1)}\left(\frac{2}{A_{1}}\right)^{p-1} 2^{p-2}\left(E(0)^{p-1}+\left(\frac{F(t)}{p}\right)^{p-1}\right)\right) \\
& =p E(0)+F(t)+\frac{p S_{2(p-1)}^{2(p-1)} 2^{2 p-4}}{A_{1}^{p-1}} E(0)^{p-1}+\frac{S_{2(p-1)}^{2(p-1)} 2^{2 p-4}}{A_{1}^{p-1} p^{p-2}} F(t)^{p-1}
\end{aligned}
$$

Since $\lim _{t \rightarrow T_{\max }^{-}} F(t)=\infty$, we can get the following lower bound of the blow-up time $T_{\max }$ from (7.2):

$$
T_{\max } \geq \int_{F(0)}^{\infty} \frac{d y}{p E(0)+y+\frac{p S_{2(p-1)}^{2(p-1)} 2^{2 p-4}}{A_{1}^{p-1}} E(0)^{p-1}+\frac{S_{2(p-1)}^{2(p-1)} 2^{2 p-4}}{A_{1}^{p-1} p^{p-2}} y^{p-1}}
$$

The proof is completed.

\section{ACKNOWLEDGMENTS}

This work was supported by the National Natural Science Foundation of China (Grant No. 11301277), the Natural Science Foundation of Jiangsu Province (Grant No. BK20151523), the Six Talent Peaks Project in Jiangsu Province (Grant No. 2015-XCL-020), and the Qing Lan Project of Jiangsu Province. 


\section{REFERENCES}

[1] M. Al-Gwaiz, V. Benci and F. Gazzola, Bending and stretching energies in a rectangular plate modeling suspension bridges, Nonlinear Anal. 106 (2014), 18-34.

[2] O. H. Ammann, T. von Karman, G. B. Woodruff, The failure of the tacoma narrows bridge, Federal Works Agency, 1941.

[3] J. M. Ball, Remarks on blow-up and nonexistence theorems for nonlinear evolution equations, Quart. J. Math. Oxford Ser. (2) 28 (1977), no. 112, 473-486.

[4] J. M. W. Brownjohn, Observations on non-linear dynamic characteristics of suspension bridges, Earthquake Engng. Struct. Dyn. 23 (1994), 1351-1367.

[5] M. M. Cavalcanti, V. N. Domingos Cavalcanti and A. Guesmia, Weak stability for coupled wave and/or Petrovsky systems with complementary frictional damping and infinite memory, J. Differential Equations 259 (2015), no. 12, $7540 \mathrm{C}-7577$.

[6] M. M. Cavalcanti, A. D. D. Cavalcanti, I. Lasiecka, X. J. Wang, Existence and sharp decay rate estimates for a von Karman system with long memory, Nonlinear Anal. Real World Appl. 22 (2015), 289-306.

[7] M. Ferhat and A. Hakem, Global existence and energy decay result for a weak viscoelastic wave equations with a dynamic boundary and nonlinear delay term, Comput. Math. Appl. 71 (2016), no. 3, 779C-804.

[8] A. Ferrero, F. Gazzola, A partially hinged rectangular plate as a model for suspension bridges, Disc. Cont. Dynam. Syst. 35 (2015), 5879-5908.

[9] A. Guesmia, Existence globale et stabilisation interne non linéaire d'un système de Petrovsky, Bull. Belg. Math. Soc. Simon Stevin 5 (1998), no. 4, 583-594.

[10] A. Guesmia, Energy decay for a damped nonlinear coupled system, J. Math. Anal. Appl. 239 (1999), no. 1, $38-48$.

[11] F. Gazzola, Nonlinearity in oscillating bridges, Electron. J. Differential Equations 2013, No. 211, 47 pp.

[12] V. Georgiev and G. Todorova, Existence of a solution of the wave equation with nonlinear damping and source terms, J. Differential Equations 109 (1994), no. 2, 295-308.

[13] A. Haraux and E. Zuazua, Decay estimates for some semilinear damped hyperbolic problems, Arch. Rational Mech. Anal. 100 (1988), no. 2, 191-206.

[14] R. Ikehata, Some remarks on the wave equations with nonlinear damping and source terms, Nonlinear Anal. 27 (1996), no. $10,1165-1175$.

[15] J.-R. Kang, Global attractor for suspension bridge equations with memory, Math. Methods Appl. Sci. 39 (2016), no. 4, $762-775$.

[16] V. Komornik, Well-posedness and decay estimates for a Petrovsky system by a semigroup approach, Acta Sci. Math. (Szeged) 60 (1995), no. 3-4, 451-466.

[17] M. Kopácková, Remarks on bounded solutions of a semilinear dissipative hyperbolic equation, Comment. Math. Univ. Carolin. 30 (1989), no. 4, 713-719.

[18] W. Lacarbonara, Nonlinear structural mechanics, Springer, New York, 2013.

[19] I. Lasiecka, S. Maad and A. Sasane, Existence and exponential decay of solutions to a quasilinear thermoelastic plate system, NoDEA Nonlinear Differential Equations Appl. 15 (2008), no. 6, 689-715.

[20] A. C. Lazer and P. J. McKenna, Large scale oscillatory behaviour in loaded asymmetic systems, Ann. Inst. H. Poincaré Anal. Non Linéaire 4 (1987), no. 3, 243-274.

[21] A. C. Lazer and P. J. McKenna, Large-amplitude periodic oscillations in suspension bridges: some new connections with nonlinear analysis, SIAM Rev. 32 (1990), no. 4, 537-578.

[22] A. C. Lazer and P. J. McKenna, Global bifurcation and a theorem of Tarantello, J. Math. Anal. Appl. 181 (1994), no. $3,648-655$.

[23] H. A. Levine, Instability and nonexistence of global solutions to nonlinear wave equations of the form $P u_{t t}=-A u+$ $\mathcal{F}(u)$, Trans. Amer. Math. Soc. 192 (1974), 1-21.

[24] H. A. Levine, Some additional remarks on the nonexistence of global solutions to nonlinear wave equations, SIAM J. Math. Anal. 5 (1974), 138-146.

[25] W. J. Liu, Y. Sun and G. Li, On decay and blow-up of solutions for a singular nonlocal viscoelastic problem with a nonlinear source term, Topological Methods in Nonlinear Analysis (2016), in press. DOI: 10.12775/TMNA.2016.077

[26] W. J. Liu and J. Yu, On decay and blow-up of the solution for a viscoelastic wave equation with boundary damping and source terms, Nonlinear Anal. 74 (2011), no. 6, 2175-2190.

[27] P. J. McKenna and W. Walter, Nonlinear oscillations in a suspension bridge, Arch. Rational Mech. Anal. 98 (1987), no. $2,167-177$.

[28] P. J. McKenna and W. Walter, Travelling waves in a suspension bridge, SIAM J. Appl. Math. 50 (1990), no. 3, $703-715$.

[29] S. A. Messaoudi, Blow up in a nonlinearly damped wave equation, Math. Nachr. 231 (2001), 105-111.

[30] S. A. Messaoudi, Global existence and nonexistence in a system of Petrovsky, J. Math. Anal. Appl. 265 (2002), no. 2, 296-308.

[31] S. A. Messaoudi, S. E. Mukiawa and E. D. Cyril, Finite dimensional global attractor for a suspension bridge problem with delay, C. R. Math. Acad. Sci. Paris 354 (2016), no. 8, 808-824.

[32] M. Nakao, A difference inequality and its application to nonlinear evolution equations, J. Math. Soc. Japan 30 (1978), no. $4,747-762$. 
[33] K. Ono, On global existence, asymptotic stability and blowing up of solutions for some degenerate non-linear wave equations of Kirchhoff type with a strong dissipation, Math. Methods Appl. Sci. 20 (1997), no. 2, 151-177.

[34] L. E. Payne and D. H. Sattinger, Saddle points and instability of nonlinear hyperbolic equations, Israel J. Math. 22 (1975), no. 3-4, 273-303.

[35] R. H. Plaut and F. M. Davis, Sudden lateral asymmetry and torsional oscillations of section models of suspension bridges, J. Sound Vib. 307 (2007), 894-905.

[36] R. Scott, in the wake of tacoma. Suspension bridges and the quest for aerodynamic stability, ASCE Press, 2001.

[37] F. Tahamtani and M. Shahrouzi, Existence and blow up of solutions to a Petrovsky equation with memory and nonlinear source term, Bound. Value Probl. 2012 (2012), no. 50, 1-15.

[38] G. Tarantello, A note on a semilinear elliptic problem, Differential Integral Equations 5 (1992), no. 3, 561-565.

[39] Y. Wang, Finite time blow-up and global solutions for fourth order damped wave equations, J. Math. Anal. Appl. 418 (2014), no. 2, 713-733.

[40] S.-T. Wu and L.-Y. Tsai, On global solutions and blow-up of solutions for a nonlinearly damped Petrovsky system, Taiwanese J. Math. 13 (2009), no. 2A, 545-558.

[41] G. Xu and J. Zhang, Existence results for some fourth-order nonlinear elliptic problems of local superlinearity and sublinearity, J. Math. Anal. Appl. 281 (2003), no. 2, 633-640.

[42] L. Yang, F. Liang and Z. H. Guo, Lower bounds for blow-up time of a nonlinear viscoelastic wave equation, Boundary. Value. Problem. 2015, 2015:219.

[43] Y. Yang and J. Zhang, Existence of solutions for some fourth-order nonlinear elliptic problems, J. Math. Anal. Appl. 351 (2009), no. 1, 128-137.

[44] Y. Ye, Global existence and blow-up of solutions for higher-order viscoelastic wave equation with a nonlinear source term, Nonlinear Anal. 112 (2015), 129-146.

[45] Y. C. You, Energy decay and exact controllability for the Petrovsky equation in a bounded domain, Adv. in Appl. Math. 11 (1990), no. 3, 372-388.

[46] J. Zhou, Lower bounds for blow-up time of two nonlinear wave equations, Appl. Math. Lett. 45 (2015), 64-68.

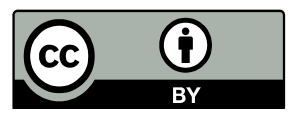

(C) 2017 by the authors; licensee Preprints, Basel, Switzerland. This article is an open access article distributed under the terms and conditions of the Creative Commons by Attribution (CC-BY) license (http://creativecommons.org/licenses/by/4.0/). 